\title{
LE DROIT A LA REPARATION DES VICTIMES DES CRIMES INTERNATIONAUX DANS LA JURISPRUDENCE DE LA COUR PENALE INTERNATIONALE
}

KENNEDY KIHANGI BINDU ${ }^{1}$

\section{ABSTRACT}

Le système du Statut de Rome de la Cour Pénale Internationale (CPI) a apporté une métamorphose fulgurante dans l'administration de la justice pénale internationale en élevant les victimes au rang de « sujet de droit international pénal ». Son succès n'est pas limité à la répression des criminels mais aussi, et surtout, à l'organisation d'une procédure de réparations (justice réparatrice) au profit des victimes des crimes internationaux relevant de la compétence de la $\mathrm{CPI}$. La jurisprudence disponible révèle des résultats mitigés et des retards inquiétants dus à des entraves d'ordre procédural et administratif. Les ordonnances de réparations semblent n'avoir pas franchi le seuil du prétoire et servent à garnir les vitrines de la Cour ainsi que celles du Fonds au profit des victimes dans son double mandat de réparation et d'assistance. La Cour a encore un long chemin à parcourir. Elle doit relever le défi et dépasser la volonté théorique de prise en compte de besoins des victimes (droit à la réparation) et atteindre la phase effective des réparations.

Mots clefs: Justice réparatrice. crimes internationaux. Cour Pénale Internationale. Fonds au profit des victimes et Statut de Rome. ordonnance de réparation.

\section{INTRODUCTION}

La création de la Cour Pénale Internationale (CPI) a été une étape décisive dans la promotion de la justice pénale internationale, du droit international humanitaire et du droit international des droits de l'homme. Elle s'est, d'une part, imposée à la conscience universelle comme une nécessité de mettre hors d'état de nuire les auteurs de crimes internationaux et la prévention de nouveaux crimes, et, d'autre part, présentée comme l'expression d'une lueur d'espoir aux victimes des crimes internationaux relevant de sa compétence (Kihangi Bindu K. : 2002, page 235). La répression de ces crimes et la reconnaissance de la justice réparatrice

1 Professeur de Droit International/Justice internationale, droits humains, environnement et ressources naturelles ; Directeur du Centre de Recherche sur la Démocratie et le Développement en Afrique, CREDDA/ULPGL ; Avocat au Barreau de Goma ; Vice-recteur honoraire de l'Université Libre des Pays des Grands Lacs, ULPGL/Goma. Mes remerciements s'adressent à mes collègues, étudiant (Junior Rex Baraka Bunani) et chercheurs à la Cour Pénale Internationale pour leurs énormes contributions à ces recherches, email : kenedybindu@gmail.com 
aux victimes, faisant bon ménage, sont une des valeurs fondamentalement incontestables du système du Statut de Rome. Deux années avant la célébration de la deuxième décennie de la $\mathrm{CPI}$, son registre des situations et affaires attire la curiosité scientifique centrée sur le régime de réparation prévu dans le Statut de Rome. La jurisprudence disponible a-t-elle rencontré le besoin d'une justice juste et équitable attachant une valeur intrinsèque à la réparation des préjudices subis par les victimes des crimes qui menacent la paix, la sécurité et le bien-être de tous (droit à la réparation) ? Les affaires Le Procureur c. Mathieu Ngudjolo Chui ; ${ }^{2}$ Le Procureur c. Thomas Lubanga ${ }^{3}$; Le Procureur C. Germain Katanga ${ }^{4}$; Le Procureur C. Jean Pierre Bemba Gombo 5 ; Le Procureur c. Ahmad Al Faqi Al Mahdi ; The Prosecutor c. William Samoei Ruto and Joshua Arap Sang ${ }^{7}$ et les ordonnances de réparation rendues sont des températures ou indices objectivement vérifiables à la portée de toute audience. Car, le succès de la Cour n'est pas seulement lié à la condamnation des criminels mais aussi, dans une certaine mesure, au succès de son système de réparation dans l'administration de la justice. ${ }^{8}$

En effet, le droit des victimes à demander réparation est un principe fondamental du droit international ${ }^{9}$ qui influe significativement la mutation observée depuis quelques décennies la justice pénale internationale. Désormais les victimes devant la CPI bénéficient des droits qui n'avaient jamais été accordés devant une juridiction pénale internationale. Elles peuvent demander, entre autres, réparation du préjudice qu'elles ont subi (Cour Pénale Internationale : page 43).

Avant l'établissement de la CPI, aucune juridiction internationale ne permettait aux victimes de demander et recevoir réparation des crimes qu'elles avaient subis. Devant le Tribunal Pénal International pour l'ex Yougoslavie (TPIY) ${ }^{10}$ et le Tribunal Pénal International pour le Rwanda (TPIR), ${ }^{11}$ les victimes n'étaient pas autorisées à demander réparation, les juges ne pouvant qu'ordonner la restitution de leurs biens. ${ }^{12}$ Ne pouvant agir que comme témoin en vue d'éclairer la religion du juge, une victime pouvait, cependant, se prémunir des jugements pour obtenir réparation devant les instances internes. Le contexte post-conflit étant généralement marqué par l'existence d'un système judiciaire en état de faillite et limité, les cours et tribunaux internes sont généralement dans l'incapacité de se prononcer sur des réparations

2 Le Procureur c. Mathieu Ngudjolo, ICC-01/04-02/12 ; ICC-PIDS-CIS-DRC-06-006/15-tFra, https://www.icc-cpi.int/CaselnformationSheets/ChuiFra.pdf, (visité le 29/05/2020).

3 Le Procureur c. Thomas Lubanga Dyilo, ICC-01/04-01/06, ICC-PIDS-DRC-01-016/17_Fra, https://www.icc-cpi.int/CaselnformationSheets/LubangaFra.pdf (visité, le 29/05/2020).

4 Le Procureur c. Germain Katanga, ICC-01/04-01/07, ICC-014/18_Fra, https://www.icc-cpi.int/CaselnformationSheets/ KatangaFra.pdf (visité le 14/04/2020).

5 Le Procureur c. Jean Pierre Bemba Gombo, ICC-01/05-01/08, ICC-PIDS-CAR-01-020/18_Fra, https://www.icc-cpi.int/ CaselnformationSheets/BembaFra.pdf, (visité le 29/05/2020).

6 Le Procureur c. Ahmad AI Faqi AI Mahdi, ICC-01/12-01/15, ICC-PIDS-CIS-MAL-01-08/16_Fra, https://www.icc-cpi.int/mali/ al-mahdi? In=fr, (visité, le 29/05/2020).

7 Le Procureur c. William Samoei Ruto et Joshua Arap Sang, ICC-01/09-01/11, ICC-PIDS-CIS-KEN-01-12/14_Fra, https:// www.icc-cpi.int/CaselnformationSheets/RutoSangFra.pdf, (visité, le 29/05/2020).

8 Le Procureur c. Thomas Lubanga Dyilo, Chambre de première instance I, Décision fixant les principes et procédures applicables en matière de réparations, daté du 7 août 2012 cité dans Le Procureur c. Germain Katanga, Ordonnance de réparation, N ${ }^{\circ}$ ICC-01/04-01/07, 24 mars 2017, para. 14 ; Le Procureur c. Thomas Lubanga Dyilo, Chambre d'appel, Ordonnance de réparation modifiée, du 3 mars 2015, traduction enregistrée le 1er août 2016, ICC-01/04-01/06-3129-AnxA-tFRA, Paragraphe 3.

9 Résolution de l'Assemblée Générale des Nations Unies, A/RES/60/147 du 16 Décembre 2005.

10 Résolution du Conseil de Sécurité des Nations Unies 827 du 25 mai 1993 créant le TPIY.

11 Résolution du Conseil de Sécurité des Nations Unies 955 le 8 novembre 1994 créant le TPIR.

12 Articles 24 al. 3 du Statut du Tribunal pénal international pour l'ex- Yougoslavie et 23 al.3 du Statut du TPIR qui disposent que «Outre l'emprisonnement du condamné, la chambre de première instance peut ordonner la restitution à leurs propriétaires légitimes de tous biens et ressources acquis par des moyens illicites, y compris par la contrainte. » 
éventuelles (Marchi S. \& Kandolo M. cité par Bakama Bope E. \& Ingange Wa ingange JD : 2015, page 198).

La victime, longtemps délaissée au profit de l'auteur du dommage, est peu à peu devenue au fil du XXème siècle un objet d'étude privilégié. Jadis, laissé au coupable qui pourrait ou pas indemniser la victime, le droit à la réparation de cette dernière s'est très vite amélioré (Schimitt : 2016, page 1). Ses besoins de soutien, d'assistance et de réparation par des fonds publics ou internationaux ont été soulignés et assimilés à des droits (Pradel J. : 2008, page 426 ; Jeangene V. : 2009, pages 7/8). Cette avancée en termes de prise en compte des droits de la victime se justifie par un certain nombre d'éléments (Marchi S. \& Kandolo M. cités par Bakama Bope E. \& Ingange wa Ingange J.D. : 2015, page 198/199 ; Pena Maria : 2013, page 251 ; Bititi Gilbert : 2011, page 293 ) :

«La pression politique exercée par les ONG ; la valorisation du statut de l'individu sur la scène internationale et l'importance croissante accordée aux droits de l'homme dans le contexte de crimes de masse. ... la prise en compte de la victime permettrait de ne plus ignorer le caractère choquant des crimes par leur violence et leurs effets sur la société tout entière. De plus, le nombre de victimes, toujours impressionnant, constitue une pression politique pour la prévention de ces crimes. Enfin, ... la victime demeure un témoin crucial pour la manifestation de la vérité, la vérité étant bien sûr la condition sine qua non si l'on veut espérer la pacification d'une société profondément meurtrie ».

Les éléments introductifs de cette réflexion (I) mettent à surface l'intention qui a animé les délégations à Rome d'accorder une attention soutenue au droit à la réparation reconnu aux victimes des crimes relevant de la compétence de la CPI. Ce droit mérite d'être circonscrit clairement dans le système du Statut de Rome (II) et présenter sans ambiguïté la ratio legis du «Fonds au profit des victimes » (III). En vue de dépasser tout sentiment d'autosatisfaction théorique du droit à la réparation des victimes des crimes relevant de la compétence de la $\mathrm{CPI}$, il sied d'interroger les recettes jurisprudentielles disponibles de la CPI (IV) avant de tirer les remarques conclusives en la matière $(\mathrm{V})$.

\section{LE DROIT A LA REPARATION DANS LE SYSTEME DU STATUT DE ROME DE LA CPI}

La théorie classique sur la responsabilité pose le principe que «tout dommage causé soit réparé par l'auteur du fait dommageable ». Trois conditions sont ici posées pour qu'un préjudice soit réparable : Le fait générateur de réparation ou la faute, le dommage ou préjudice et le lien de causalité ou l'exigence de la cause à effet. Tout en considérant la nécessité du préjudice pour qu'il y ait responsabilité civile, la faute apparaît ici comme un élément constitutif de la responsabilité à établir. En matière des crimes internationaux, l'auteur de la faute ne peut réparer que lorsque l'acte criminel qu'il a posé a causé préjudice à autrui. Tel est le cas de l'affaire Le Procureur c. Ahmad Al Faqi Al Mahdi, l'accusé reconnaît " avoir le remord des préjudices causés à sa famille, à sa communauté à Tombouctou, à son pays et à la communauté internationale ... et fait la promesse solennelle que la faute qu'il a commise 
envers eux sera la première et la dernière ${ }^{13}{ }^{13}$ Ce qui traduit l'expression de la faute intentionnelle commise par ce dernier et l'oblige à réparer.

La notion de la faute a été fortement critiquée par différents doctrinaires suite à ses limites pour engager la responsabilité civile de son auteur. Vers la fin du XIXème siècle, Saleilles et Josserand vont, ainsi, proposer la théorie de la responsabilité civile objective qui repose sur la notion du risque créé et le risque profit (Mubalama Zibona : 2015). La théorie du risque viserait seulement qu'aucune victime ne reste sans être indemnisée (Savatier R. : 2000, page 45). Tout en prenant acte de cette évolution doctrinale, « La faute était maintenue comme condition et fondement de la responsabilité civile. Là est le principe : pas de responsabilité civile sans faute. On peut cependant admettre que, dans certaines conditions, il soit urgent de secourir les victimes en établissant une responsabilité en dehors de toute faute " (Geneviève V. : 1965, page 12 ; Starck Boris : 1958, page 475).

Dans un autre registre, Boris Stack (1958, page 475) ${ }^{14}$ va s'appesantir sur la théorie de la garantie comme une solution idoine à l'antagonisme des pensées des théories de la faute et du risque. La victime retrouve ici sa place car désormais l'attention n'est plus portée uniquement du côté de l'auteur du dommage. La victime a des droits qui doivent être garantis notamment le droit à la réparation de toute atteinte à sa personne ou à son patrimoine. Dans le cas d'espèce, il n'y a pas de faute à exiger du responsable, la faute qui, jusque-là, permettait l'identification du responsable était devenue un obstacle à l'indemnisation de la victime. Car, si la victime n'arrivait pas à prouver la faute, elle se retrouvait sans réparation. Au final, le domaine de la responsabilité subjective s'est considérablement restreint au profit des cas de responsabilité objective qui continuent à se multiplier (Kangulumba Mbambi : 2002). Aujourd'hui, c'est bien plus le dommage qui engendre la responsabilité civile que le comportement du responsable. II va alors apparaitre le souci corollaire de ne plus faire peser le poids de la réparation sur les seuls responsables. On va se mettre à assister au déclin de la responsabilité individuelle parallèlement à la socialisation des risques. C'est la thèse que défend Geneviève V. (1965, page 12). Cette socialisation des risques a contribué à répartir le poids de l'indemnisation des dommages sur la collectivité tout entière, elle s'est mise en place par les mécanismes de l'assurance et de la socialisation des risques. Ainsi, parmi les mécanismes de socialisation des risques, on épingle le fonds de garantie (ou indemnisation) qui en vue d'éviter que les victimes ne supportent le poids de l'insolvabilité du responsable (lorsque le responsable n'est pas assuré ou lorsqu'il n'est pas identifié, ou lorsque quoiqu'identifié, le responsable n'est pas en mesure de supporter le poids de la réparation); et la sécurité sociale qui est une expression et un symbole de la solidarité sociale en matière de responsabilité civile. L'idée derrière cette grande réflexion est de renforcer la responsabilité objective, une responsabilité non attachée à la faute. La protection de la victime a désormais pris une ampleur significative en garantissant son indemnisation. ${ }^{15}$ "Pas de préjudice, pas d'actions " (Letourneau P. : 1982, page 156) sans dommage, pas de réparation, le préjudice ou le dommage est une des conditions substantielles pour engager la responsabilité de quelqu'un. Cependant, tous les dommages que suscite la vie en société ne donnent pas lieu à réparation. Toute réparation exige que le dommage ait un caractère certain (Esmein V. : 1962, page

13 Affaire Le Procureur c. Ahmad Al Faqi Al Mahdi, N ICC-01/12-01/15, 27 septembre 2016, Paragraphe 103.

14 La responsabilité fondée sur le risque et le garantie, cours de droit.net, https://cours-de-droit.net/la-responsabilite-fondee-sur-le-risque-et-la-garantie-a126822778/ 
151 ; Boré J. : 1974, page 34), direct (Terré F. : 1999, page 635 ; Montanier J. : 1981, page 45 ; Nguyen J. : 1976, page 1), légitime de l'intérêt (Vidal J. : 1971, page 23), et ne doit avoir été déjà réparé (Vidal J. : 1980, page 23).

Répondant aux caractéristiques sus présentées, on enregistre plusieurs sortes de dommages réparables en matière des crimes internationaux. Les victimes des crimes internationaux (crimes contre l'humanité, crimes de guerre, crimes de génocide et crime d'agression) peuvent avoir subi des atteintes touchant leurs corps, leurs patrimoines (Guidon M. : 2006, page 45$)^{16}$ ou encore à apprécier sur le plan moral comme l'affirme Nyabirungu R. (2013, page 8) : «A l'occasion des événements tragiques qui ont bouleversé le monde (...), les victimes ont subi plusieurs sortes des dommages tels les viols, pillages, destructions des champs et des récoltes, la démolition des maisons que la paisible population a subi, sont des dommages physiques, moraux ou matériels et nécessitent réparation pour établir une justice et une paix sociale entre les humains ". Le dommage corporel est, d'abord et avant tout, toute atteinte portée à l'intégrité physique de la personne : les blessures plus ou moins graves et à plus forte raison la mort. Ces dommages appellent, bien entendu, à l'indemnisation de la victime. Mieux vaut dire indemnisation que réparation, car on ne ressuscite pas les morts, et il est malaisé, c'est le moins qu'on puisse dire, de rendre à l'amputé son bras ou sa jambe (Terré F. : 1999, page 640 ; Assale C. : 1990, page 12 ; Lambert Y. : 1990, page 5 ; Gasigwa H. : 2000, page 34). La réparation du préjudice est due même si la victime est tombée dans un état de totale inconscience. Pour le préjudice corporel, la notion semble être trop étendue car relève aussi de la catégorie des dommages corporels, des souffrances physiques, passées ou futures, subies par la victime (Guidon M. : 2006, page 45). Le préjudice matériel est celui qui se traduit par une perte évaluable pécuniairement, il s'agit d'un préjudice patrimonial (Chabas F. :1965, page 405).

Le préjudice moral, en revanche, ne se traduit point par une perte en argent, par ce qu'il porte atteinte à un droit extrapatrimonial. ${ }^{17}$ Lorsque le préjudice subi cesse d'être corporel ou matériel et revêt un caractère extrapatrimonial, sa réparation peut susciter des objections, soit d'une manière générale, parce qu'il est alors singulièrement difficile d'aménager une réparation adéquate, soit de manière plus particulière, lorsqu'il s'agit d'une douleur morale car il peut être choquant d'aller en quelque sorte monnayer ses larmes devant les tribunaux (Ripert G. : 1948, page 3). Une lecture fouinée de l'affaire Katanga révèle que les victimes ont affirmé avoir subi des préjudices moraux résultant de la perte des décès des parents et d'autres proches. Elles ont rappelé les traumatismes, les stress moraux et cauchemars auxquels elles sont exposées toutes les fois qu'elles pensent aux êtres chers perdus. ${ }^{18}$ En considération de ces allégations, le représentant légal des victimes notera que l'indemnisation du préjudice moral doit tenir compte de trois catégories des victimes :

«En premier lieu, les parents très proches (père, mère, époux, enfants et assimilés) ; en second lieu, les parents proches (frères, sœurs, et assimilés) ; en troisième lieu, les autres parents plus éloignés. Pour la première catégorie, il suggère une fourchette de 25.000 à 50.000 USD, pour la seconde, une fourchette de 12.500 à 25.000 USD, pour la troisième, une fourchette de 6.000

16 Affaire Le Procureur c. Ahmad Al Faqi Al Mahdi, N ICC-01/12-01/15, 27 septembre 2016, Paragraphe 103.

17 Le Procureur c. Germain Katanga, Ordonnance de réparation en vertu de l'article 75 du Statut de Rome, N ICC-01/04-01/07, Paragraphe 239 
à 12.000 USD $» .{ }^{19}$ Partageant la même lecture, la Défense a aussi suggéré pour « la première catégorie une somme de 10.000 USD, pour la seconde une somme de 6.000 USD et pour la dernière un montant de 4.000 USD ».

C'est en référence aux pratiques observées en France, en Belgique, en RDC (juridictions militaires), devant la Commission des Nations Unies en matière d'indemnisation et devant la Cour Interaméricaine que la Chambre de première instance II de la CPI va retenir deux catégories de décès ayant un impact sur chacune des victimes : "D'une part, des parents proches (conjoints, parents, enfants, grands-parents, petits-enfants), et, d'autre part, celui des autres parents plus éloignés (autres parents). Le préjudice psychologique lié au décès d'un parent proche est évalué ex æquo et bono à 8.000 USD et le préjudice psychologique lié au décès d'un parent plus éloigné est évalué ex æquo et bono à 4.000 USD $"{ }^{20}$ La chambre a particulièrement pris en considération l'affaire Puerto Bello massacre v. Colombia ${ }^{21}$, dans laquelle la Cour Interaméricaine :

« ... réaffirme que la souffrance causée à une victime concerne les membres de la famille les plus proches, particulièrement ceux qui avaient des relations affectives proches avec la victime. De plus, la (Cour Interaméricaine) a présumé que les souffrances ou la mort d'une personne entraine pour ses enfants, son époux ou son compagnon, sa mère, son père ou ses enfants un préjudice non-pécuniaire qui n'a pas besoin d'être prouvé $»{ }^{22}$

La réparation des dommages n'est pas subordonnée uniquement à la double existence d'un dommage (matériel, corporel ou moral) et d'un fait générateur de responsabilité (fait personnel) ; encore faut-il que ce dommage se rattache à ce fait générateur de responsabilité par un lien de causalité (Marty G. : 1939, page 685 ; Chabas F. : 1965, page 70). II faut que le fait générateur de responsabilité ait été la cause du dommage, sa cause efficiente. Dans l'affaire Katanga, la Chambre d'appel II a jugé que « la norme applicable au lien de causalité entre le préjudice et le crime est le critère dit du «but/for » en common law à savoir que n'eût été la commission du crime, le préjudice n'aurait pas été constitué, et il est en outre requis que les crimes dont Thomas Lubanga a été reconnue coupable aient été la « cause directe » du préjudice pour lequel des réparations sont demandées $»{ }^{23}$

Le droit à la réparation est protégé par les articles 75 et 79 du Statut de Rome de la CPI et la Résolution 60/147 adoptée par l'Assemblée Générale des NU du 16 décembre 2005. La Cour établit des principes applicables aux formes de réparation, telles que la restitution, I'indemnisation ou la réhabilitation, à accorder aux victimes ou à leurs ayants droit. Une réparation adéquate, effective et rapide permet de promouvoir la justice en remédiant aux violations flagrantes du droit international des droits de l'homme ou aux violations graves du droit international humanitaire.

Dans son étendue, le droit à la réparation en matière des crimes internationaux conduit à l'idée de la réparation intégrale (Fofe Djofia Malewa : 1998, pages 31/48). Selon Viney et

19 Le Procureur c. Germain Katanga, Ordonnance de réparation en vertu de l'article 75 du Statut de Rome, N ICC-01/04-01/07, Paragraphe 239, P. 227.

20 Le Procureur c. Germain Katanga, Ordonnance de réparation en vertu de l'article 75 du Statut de Rome, N ICC-01/04-01/07, Paragraphe 230-232.

21 Cour Interaméricaine, Puerto Bello Massacre v. Colombia (Merits, Reparations and Costs), Paragraphe 257.

22 Le Procureur c. Germain Katanga, Ordonnance de réparation en vertu de l'article 75 du Statut de Rome, N ICC-01/04-01/07, Paragraphe 231.

23 Le Procureur c. Thomas Lubanga, Chambre d'appel, Ordonnance de réparation, n01/04-01/06-3129-AnxA-tFRA, Paragraphe 59. 
Jourdain, le principe de la réparation intégrale est annoncé par une formule classique, quasi dogmatique, selon laquelle :

Le propre de la responsabilité civile est de rétablir aussi exactement que possible l'équilibre détruit par le dommage et de replacer la victime dans la situation où elle se serait trouvée si l'acte dommageable n'avait pas eu lieu (Mestre G. : 2005, page 161 ; Telomono M. : 2014, page 380). Le dogme de la réparation intégrale est souvent résumé par l'expression « Rien que le dommage, tout le dommage » (Geneviève V. : \& Jourdain 2010, page 154).

De cette finalité, la doctrine déduit habituellement deux conséquences : d'une part, la victime ne doit pas s'enrichir du fait de la réparation. D'autre part, la réparation doit porter sur la totalité des préjudices subis, nonobstant les difficultés d'évaluation qui pourraient éventuellement surgir (Geneviève V. : \& Jourdain 2010, page 154). Telle est aussi la position de la jurisprudence Française selon laquelle « les dommages-intérêts alloués à une victime doivent réparer le préjudice subi sans qu'il en résulte pour elle ni perte, ni profit ». ${ }^{24}$

Si l'idéal est que le préjudice soit réparé intégralement, il faut avouer qu'en certaines matières, la réparation intégrale se bute à une difficulté d'être appliquée. Souvent, le juge ne sait pas apprécier intégralement le préjudice subi, qu'il recourt à l'équité pour allouer les indemnités à la victime. Matthieu Telomono (2014, page 23) soutient qu'en cas de la mort d'un être cher ou du préjudice moral subi résultant de la mort du conjoint, de l'ami, aucune réparation intégrale ne peut être conçue car il est impossible de ramener à la vie la personne morte. En matière particulière des crimes internationaux et des violences sexuelles, focalisant l'attention sur la situation de la RDC, tantôt les juridictions accordent des dommages-intérêts de manière superficielle et sans motivation quelconque, tantôt, elles prononcent les dommages intérêts qui reflètent les préjudices subis. Tout cela dépend des humeurs et des attitudes des juges. Il a été observé, par exemple, en RDC, en matière des violences sexuelles et de crimes contre la paix et la sécurité de l'humanité, la Cour d'Appel du Haut Katanga a, dans deux affaires différentes, faisant application de l'équité et du bon sens, accordé respectivement à titre de réparation sous le RPA 6301/010 un pagne premier choix, un costume, les souliers pour papa et maman, un sac à main et deux chèvres tandis que sous le RPA 6298/010, 10.000 USD. Visiblement, la valeur pécuniaire d'un cas des violences sexuelles et de crime contre la paix et la sécurité de l'humanité (crime international) en termes de dommages intérêts ne suit aucun barème préétabli (PNUD : 2018, pages 5\&45).

Quelques hauts magistrats réunis à Lubumbashi en RDC en atelier, le 7 juillet 2018, ont affirmé que : "Même si la victime postule pour les dommages-intérêts, le juge considère aussi la coutume locale en termes d'exigences relativement au versement de la dot pour déterminer le taux des dommages intérêts à prononcer ». Toutes considérations faites, il n'y a aucune constance dans les allocations qui sont faites par les juridictions civiles et militaires en RDC PNUD : 2018, pages 5 \& 45).

Cette réflexion n'est pas éloignée des pratiques auxquelles la Chambre de première instance I dans l'affaire Katanga a fait recours dans l'appréciation des dommages-intérêts à allouer aux victimes des crimes graves. ${ }^{25}$ Dans certaines circonstances où le juge ne sait pas apprécier la gravité du préjudice subi par les victimes, il peut être amené à procéder unique-

24 Cassation civile française 2ème, 5 Juillet 2005, Bulletin civil II $n^{\circ} 4$. 
ment à des réparations collectives (article 97 du Règlement de procédure et de preuve de la $\mathrm{CPI})$. L'inconvénient des réparations collectives demeurant le fait que la réparation ne tient pas compte de l'évaluation vraie du préjudice que chacune des victimes a subi. Le préjudice enduré n'étant pas le même ou le degré de souffrance étant différent, l'idéal serait que la réparation en matière des crimes internationaux soit individualisée. C'est en veillant sur le sort des victimes qui doivent bénéficier des réparations, que le Statut de Rome a institué le Fonds au profit des victimes.

\section{PRESENTATION ET PORTEE JURIDIQUE DU FONDS AU PROFIT DES VICTIMES CONSACRE PAR L'ARTICLE 79 DU STATUT DE ROME}

Contrairement aux tribunaux militaires internationaux (Nuremberg et de Tokyo) et aux tribunaux ad hoc (TPIY et TPIR), la CPI a apporté une métamorphose fulgurante dans l'administration de la justice pénale internationale quant à ce qui est du sort réservé aux victimes devant elle. Le système du Statut de Rome et celui des juridictions pénales "internationalisées" ou tribunaux mixtes créés dans sa suite (particulièrement les Chambres extraordinaires au sein des tribunaux Cambodgiens et du Tribunal spécial pour le Liban, TSL) élèvent les victimes au rang ou au statut de " sujet de droit international pénal ». Ce passage marque une « prise de conscience accrue en droit international pénal de la nécessité de dépasser la notion de justice punitive, pour tendre vers une solution plus inclusive, qui encourage les victimes à participer au processus et reconnaît le besoin de leur offrir des recours utiles. $»^{26}$

Cette dynamique portée par l'état d'esprit des délégations réunies à la conférence diplomatique à Rome « ... des millions d'enfants, de femmes et d'hommes ont été victimes d'atrocités qui défient l'imagination et heurtent profondément la conscience humaine " a conduit à la création de deux institutions indépendantes : la CPI et le Fonds au profit des victimes (Tshibuyi wa Tshibuyi : 2009 ; Kihangi Bindu K. : 2010 ; 2015, page 162).

L'article 79 du Statut de Rome esquisse les contours du Fonds tout en laissant à l'Assemblée des Etats Parties le soin de fixer les principes de sa gestion. Il est un instrument de la Cour (mandat de réparation) et pose des actions de manière largement indépendante des activités judiciaires de la Cour (assistance aux victimes). Selon la Cour, « les réparations ont deux objectifs principaux consacrés par le Statut : elles obligent les responsables de crimes graves à réparer le préjudice qu'ils ont causé aux victimes et elles permettent à la Cour de s'assurer que les criminels répondent de leurs actes ${ }^{2}{ }^{27}$

La structure du Fonds au profit des victimes est déterminée par les articles 1 et 17 du Règlement du Fonds d'affectation spéciale au profit des victimes. ${ }^{28}$ L'analyse de ces disposi-

26 Préambule du Statut de Rome de la CPI ; Le Procureur c. Thomas Lubanga Dyilo, Chambre d'appel, Ordonnance de réparation (modifiée), 3 mars 2015, traduction enregistrée le 1 août 2016, ICC-01/04-01/06-3129AnxA Paragraphe 1.

27 Le Procureur c. Thomas Lubanga Dyilo, Chambre d'appel, Ordonnance de réparation (modifiée), 3 mars 2015, traduction enregistrée le 1 août 2016, ICC-01/04-01/06-3129AnxA, paragraphe 2 .

28 ICC-ASP/1/Res. 6 portant création d'un fonds au profit des victimes de crimes relevant de la compétence de la Cour et de leurs familles, adoptée par consensus, à la séance plénière, le 9 septembre 2002, paragraphes $1-7$. 
tions débouche sur le fait que le Fonds au profit des victimes a deux organes qui assurent sa gestion : Le Conseil de direction et le Secrétariat du Fonds au profit des victime. ${ }^{29}$ Le Fonds au profit des victimes assure les fonctions de réparation et d'assistance au profit des victimes. Ce Fonds peut agir dans l'intérêt des victimes de crimes, que soit intervenue ou non une condamnation par la CPI. II coopère avec la Cour afin d'éviter toute interférence dans les procédures judiciaires en cours (Cour Pénale Internationale : 2011).

Le pouvoir de gestion du Fonds est confié au Conseil de direction, il dispose d'un financement propre, distinct du budget général de la Cour. Les points 22, 23 et 25 du Règlement du Fonds d'affectation spéciale au profit des victimes précisent respectivement que

«Dans son rapport annuel à l'Assemblée des États Parties sur les activités et projets du Fonds, le Conseil de direction soumet un appel de contributions volontaires au Fonds.

Avec l'appui du Secrétariat, le Conseil de direction prend contact avec les gouvernements, les organisations internationales, les particuliers, les entreprises et d'autres entités afin de solliciter des contributions volontaires au Fonds.

Le Fonds reçoit toutes les contributions volontaires versées par les sources citées à I'alinéa a) du paragraphe 2 de la résolution ICC-ASP/1/Res. 6 de I'Assemblée des États Parties et prend note des sources et des montants reçus " ${ }^{30}$

Bien que fonctionnant avec des contributions volontaires, le Fonds a la latitude à pouvoir refuser certaines contributions. La Règle 30 du Règlement du Fonds d'affectation spéciale au profit des victimes est précis en ces termes:

Le Fonds refuse les contributions volontaires :

a) Considérées comme n'étant pas compatibles avec les buts et les activités du Fonds ;

b) Considérées comme étant affectées à une destination d'une manière incompatible avec la règle 27 (avant de refuser de telles contributions, le Conseil de direction peut s'efforcer d'obtenir du donateur qu'il renonce à cette destination ou qu'il la modifie dans un sens qui soit acceptable);

c) Qui affecteraient l'indépendance du Fonds ;

d) Qui entraîneraient une répartition manifestement inéquitable des ressources et biens disponibles entre les différents groupes de victimes.

Janet Chan (2013, page 12) précise que le Fonds au profit des victimes est une institution non judiciaire et indépendante de la CPI. II administre, d'une part, les ordonnances de réparation de la $\mathrm{CPI}$ lorsqu'un accusé est déclaré coupable, et, d'autre part, fournit de l'assistance générale aux victimes et à leurs familles grâce aux contributions volontaires. Par son financement et projets, il tente de répondre à leurs besoins physiques, psychologiques et matériels. II faut noter que le Fonds peut exercer son mandat d'assistance même en l'absence d'une condamnation par la CPI.

29 ICC-ASP/3/Res7, Création du Secrétariat du fonds d'affectation spéciale au profit des victimes, adoptée le 10 ; Septembre 2004 ; articles 18 et 19 du Règlement du Fonds au profit des victimes. décembre 2005. 
La phase des réparations apparaît ici comme une étape essentielle de l'administration de la justice car le succès de la Cour, dans une certaine mesure, est lié au succès de son système de réparation. ${ }^{31}$ En vue de présenter la valeur intrinsèque de cette deuxième institution créée par le Statut de Rome "Fonds au profit des victime », la Chambre de Première Instance II dans l'Affaire Katanga affirme sans ambages que:

«La procédure en réparation est à la fois liée à la procédure pénale et séparée de cette dernière. Elle est liée à la procédure pénale, car la responsabilité en matière de réparations est étroitement liée aux crimes pour lesquels la personne a été reconnue coupable. Elle est séparée de cette procédure, car elle constitue une procédure en soi, dans le cadre de laquelle des preuves spécifiques sont produites par les victimes qui peuvent être, lorsque cela est possible et avec les expurgations qu'il convient d'appliquer, contestées par la personne reconnue coupable. A cette occasion, des observations et des arguments oraux et écrits sont échangés par les parties sur les différents aspects juridiques et factuels de la procédure. L'ensemble de ces échanges trouve son aboutissement dans l'ordonnance de réparation ... comme toute procédure devant la Cour, la phase des réparations est une procédure judiciaire. Partant, la Chambre doit assurer un juste équilibre entre les droits et intérêts divergents des victimes et ceux de la personne déclarée coupable ". 32

Abordant l'aspect d'assistance, Motoo Noguchi, Président du Conseil de direction du Fonds au profit des victimes lors de la séance de la 12ème session de l'Assemblée des Etats parties de la CPI a salué le rôle joué par le Fonds dans son mandat d'assistance dans le Nord de l'Ouganda et en RDC depuis 2008. ${ }^{33}$ John Bolton note que « depuis 2008, le Fonds a apporté son aide à plus de 450.000 victimes directes et indirectes en RDC et en Ouganda, en assurant une réadaptation physique et psychologique ainsi qu'un soutien socioéconomique aux survivants des crimes les plus graves. Le Fonds est également au stade de la mise en œuvre des trois ordonnances de réparation dans les affaires Lubanga et Katanga en RDC et

31 Le Procureur c. Germain Katanga, Chambre de Première Instance II, Ordonnance de réparation, ICC-01/04-01/07-3728 du 24 mars 2017, Paragraphe 14 ; Le Procureur c. Thomas Lubanga Dyilo, Chambre de première instance I, Décision fixant les principes et procédures applicables en matière de réparations, daté du 7 août 2012 et traduction enregistrée le 19 février 2013, ICC-01/04-01/06-2904-tFRA, Paragraphe 178 (" Lubanga, Chambre de Première Instance I, Décision sur les réparations ") ; Le Procureur c. Thomas Lubanga Dyilo, Chambre d'appel, Ordonnance de réparation (modifiée), 3 mars 2015, traduction enregistrée le 1 août 2016, ICC-01/04-01/06-3129AnxA, paragraphe 3 (Lubanga, Chambre d'appel, Ordonnance de réparation, ICC-01/04-01/06-3129AnxA-tFRA »).

32 Le Procureur c. Germain Katanga, Chambre de Première Instance II, Ordonnance de réparation, ICC-01/04-01/07-3728 du 24 mars 2017, paragraphes 16 \& 18 ; Le Procureur c. Thomas Lubanga Dyilo, Chambre d'appel, Decision on the admissibility of the appeals against Trial Chamber l's " Decision establishing the principles and procedures to be applied to reparations " and directions on the further conduct of proceedings, 14 décembre 2013, ICC-01/04-01/06-2953, paragraphe $70:$ : the reparations proceedings are a distinct stage of the proceedings and it is conceivable that different evidentiary standards and procedural rules apply to the question of who is a victim for the purposes of those proceedings "; Lubanga, Chambre d'appel, Arrêt sur les réparations, ICC-01/04-01/06-3129, Paragraphe 237 : « the Appeals Chamber considers it to be beyond question that a person subject to an order of court of law must know the precise extent of his or her obligations arising from that court order, particularly in light of the corresponding right to effective appeal such an order, and that the exten of those obligations must be determined by a court in a judicial process "; Lubanga, Chamber d'appel, Ordonnance de réparation, ICC_01/04_01/06-3129-Anx-tFRA, Paragraphes 20, 22, 45 et 49. Ces principes applicables aux réparations ne sauraient être interprétés de façon préjudiciable ou contraire aux droits de la personne déclarées coupable et aux exigences d'un procès équitable et impartial ; Article 97-3 du Règlement de Procédure et de Preuve de la CPI.

33 M. Motoo Noguchi, Rapport à l'Assemblée des Etats parties sur les activités et les projets du conseil de direction du Fonds d'affectation spéciale au profit des victimes pour la période du 1er juillet 2012 au 30 Juin 2013, Paragraphes 39 et 46, https://asp.icc-cpi.int/iccdocs/asp_docs/ASP15/ICC-ASP-15-14-FRA.pdf ; Fonds au profit des victimes, Assistance et réparations, réalisations, enseignements tirés et évolution, Rapport sur l'état d'avancement des programmes, Septembre 2015. 
dans l'affaire Al Mahdi au Mali, où plus de dix mille victimes bénéficieront des programmes de réparations ${ }^{34}$.

En tant qu'innovation pionnière spécifique à la $\mathrm{CPI}$, le Fonds au profit des victimes doit intervenir au profit des victimes dans les limites établies des ordonnances de réparation ou de son programme d'assistance aux victimes. Il s'agit ici d'une mise en œuvre effective du droit à la réparation reconnue aux victimes en droit international des droits de l'homme et en droit international humanitaire.

\section{COMPRENDRE LE DROIT A LA REPARATION DANS LA JURISPRUDENCE DE LA CPI}

Depuis l'entrée en vigueur en 2002 du Statut de Rome de la CPI adoptée en 1998, le nombre des situations et des affaires n'a cessé d'augmenter. Sans vouloir verser ou alimenter les controverses et reproches variés faits à l'endroit de la CPI sur différents aspects notamment les durées trop longues d'instruction des affaires, les critères de sélection des situations et affaires selon les zones géographiques, l'éventuelle instrumentalisation de la Cour par certaines puissances Etatiques, la politique de poursuite de chefs d'Etats Africains en fonction, d'aucuns notent des efforts notables de la Cour en faveur des victimes. La jurisprudence disponible au stade actuel fait école quant aux réparations et assistances accordées aux victimes des crimes relevant de sa compétence.

\section{LES RÉPARATIONS DANS L'AFFAIRE LUBANGA}

Après avoir déclaré Thomas Lubanga Dyilo coupable des crimes de conscription et d'enrôlement d'enfants de moins de 15 ans au sein de l'Union des Patriotes Congolais «UPC » et des Forces Patriotiques pour la Libération du Congo «FPLC » et du fait de les avoir fait participer activement à des hostilités, au sens des articles 8-2-e-vii et 25-3-a du Statut, de septembre 2002 à août 2003 en en Ituri/RDC, ${ }^{35}$ la Chambre a tenu d'autres audiences sur la peine et les réparations conformément aux articles 76-2 du Statut et 143 du RPP. Les audiences des réparations ont connu un parcours riche en termes des principes et procédures applicables aux réparations et au plan de mise en œuvre des réparations accordées aux victimes :

Le Procureur $c$. Thomas Lubanga, Chambre de Première Instance I, Décision Fixant les principes et procédures applicables en matière de réparations, ICC-01/04-01/06 du 7 août 2012, ICC-01/04-01/06-2904-tFRA, le 19 février 2013.

Le Procureur c. Thomas Lubanga Dyilo, Chambre d'appel, Ordonnance de réparation modifiée, du 3 mars 2015, ICC-01/04-01/06-3129-AnxA-tFRA, le 1 er août 2016.

34 Le Conseil de direction du Fonds au profit des victimes affirme que la justice réparatrice prévue par le Statut de Rome revêt une importance incontestable pour les victimes, Déclaration du 14 Septembre 2018, https://www.icc-cpi.int/Pages/item. aspx? name=180914-stat-tfv\&ln=fr, (visité le 5 mai 2020).

35 Le Procureur c. Thomas Lubanga Dyilo, ICC-01/04-01/06 du 14 mars 2012, ICC-01/04-01/06-2842-tFRA 31/08/2012, paragraphe 1358. 
Le Procureur c. Thomas Lubanga Dyilo, Chambre de Première Instance II, Rectificatif de la « Décision fixant le montant des réparations auxquelles Thomas Lubanga Dyilo est tenu ", version publique expurgée, ICC-01/04-01/06 du 21 décembre 2017, ICC-01/04-01/06-3379-Red-Corr du 21 décembre 2017.

Par ces ordonnances, la Cour rappelle la portée des réparations en insistant sur le fait qu'il est du droit des victimes de demander et d'obtenir réparation. Ce droit a déjà trouvé une consécration importante sur le plan international. ${ }^{36}$ En cela, la Cour a la latitude d'accorder aux victimes une réparation individuelle ou collective et, lorsqu'elle l'estime appropriée, elle peut accorder les deux concurremment car elles ne s'excluent pas mutuellement. ${ }^{37}$ Les réparation individuelles à accorder ne doivent, cependant pas, constituer une source des tensions et de divisions au sein des communautés. Lorsqu'il est envisagé des réparations collectives, on doit s'assurer qu'elles remédient au préjudice que les victimes ont subi aussi bien individuellement que collectivement. ${ }^{38}$

Cette première décision de la $\mathrm{CPI}$ en matière de réparations, accueillie dans différents milieux notamment diplomatiques, de la société civile et du savoir, apparaît comme une innovation notable en matière de réparations au profit des victimes des crimes internationaux. Elle était cependant entachée ou teintée de certaines faiblesses ayant conduit la Chambre d'appel au devoir de rappeler ce qu'une ordonnance de réparation se doit de contenir :

- être prise à l'encontre de la personne condamnée, Mr Lubanga le cas échéant ;

- établir la responsabilité financière de Mr Lubanga et l'en informer, ainsi que du fait que, exceptionnellement, le Fonds d'aide au profit des victimes se chargeait de son évaluation ;

- stipuler les types de réparation - individuelle et/ou collective - qui sont octroyés ;

- définir les types de préjudice qui peuvent faire l'objet d'une réparation, en gardant à l'esprit la nécessité d'un lien entre le préjudice subi et les crimes pour lesquels Mr Lubanga a été condamné ;

- Identifier les victimes susceptibles d'être éligibles à la réparation ou énoncer les critères d'éligibilité (Redress Trust : 2017, pages 6 \& 7).

Il a fallu attendre le rectificatif de la «Décision fixant le montant des réparations auxquelles Thomas Lubanga Dyilo est tenu » de la Chambre de première instance II, du 21 décembre 2017, dans sa traduction enregistrée du 21 décembre 2017 pour qu'elle fixe le montant des réparations, le nombre des victimes, déclare son indigence et enjoint au Conseil du Fonds d'indiquer s'il est en mesure d'affecter un montant supplémentaire à la mise en œuvre des réparations collectives dans la présente affaire en ces termes :

« Rend, à l'unanimité, la présente Décision fixant le montant des réparations auxquelles $\mathrm{M}$. Thomas Lubanga est tenu ;

36 Résolution de l'Assemblée Générale des Nations Unies, A/RES/60/147 du 16 Décembre 2005, voir le texte en annexe du chapitre I, Evolution de l'accès des victimes à la justice.

37 Le Procureur c. Thomas Lubanga, Chambre de Première Instance I, Décision Fixant les principes et procédures applicables en matière de réparations, ICC-01/04-01/06-2904-tFRA du 7 août 2012 et traduction enregistrée le 19 février 2013, paragraphes $217-221$.

38 Le Procureur c. Thomas Lubanga Dyilo, Chambre d'appel, Ordonnance de réparation modifiée, du 3 mars 2015, ICC-01/0401/06-3129-AnxA-tFRA, le 1er août 2016, paragraphe 33. 
Constate que 425 des 473 victimes potentiellement éligibles issues de l'échantillon ont démontré au standard de preuve de l'hypothèse la plus probable être une victime directe ou une victime indirecte des crimes pour lesquels M. Lubanga a été déclaré coupable ;

Décide, par conséquent, que les 425 victimes doivent bénéficier des réparations collectives approuvées par la Chambre dans la présente affaire ;

Constate que les 425 victimes ne constituent qu'un échantillon de victimes potentiellement éligibles et que des centaines voire des milliers de victimes additionnelles ont subi de préjudice résultant des crimes pour lesquels $M$. Lubanga a été condamné ;

Fixe le montant des réparations auxquelles $\mathrm{M}$. Lubanga est tenu à la somme totale de 10.000.000 USD, ce qui comprend à la fois sa responsabilité à l'égard des 425 victimes issues de l'échantillon, soit 3.400.000 USD, et sa responsabilité à l'égard des autres victimes qui pourraient être identifiées, soit 6.600.000 USD ;

Déclare que M. Lubanga est indigent aux fins des réparations au jour de la présente Décision ;

Enjoint au Conseil de direction du Fonds de lui indiquer s'il est en mesure d'affecter un montant supplémentaire à la mise en œuvre des réparations collectives dans la présente affaire, dans le respect des dispositions de la règle 56 du Règlement du Fonds, ou de poursuivre ses efforts visant la collecte de fonds supplémentaires, au plus tard le 15 février 2018;

Enjoint à la Présidence, avec l'assistance du Greffier, de surveiller de manière continue la situation financière de $\mathrm{M}$. Lubanga conformément à la norme 117 du Règlement de la Cour ;

Enjoint au Fonds de prendre contact avec le Gouvernement de la RDC en vue d'établir la manière dont il pourrait contribuer au processus des réparations et tenir la Chambre informée à ce sujet ;

Enjoint au Fonds de déposer des observations sur la possibilité de poursuivre la recherche et l'identification des victimes avec l'assistance du BCPV et des Représentants légaux des victimes V01 et V02, au plus tard le 15 janvier 2018 ;

Décidera sur la suite de la mise en œuvre des réparations collectives en temps opportun ;

Invite le Fonds à envisager la possibilité d'inclure les personnes qui ne remplissent pas le critère requis afin de bénéficier des réparations ordonnées dans la présente affaire dans les programmes d'assistance mis en place dans la zone de situation en RDC ;

Enjoint au Greffier de prendre toutes les mesures nécessaires pour donner une publicité adéquate à la présente décision ${ }^{39}{ }^{39}$

Près de huit ans après, depuis le déclenchement de la procédure de réparations dans l'affaire Lubanga, est-il encore permis de croire à son aboutissement au profit des victimes? Les victimes craignent pour l'effectivité de la réparation suite à la libération de Lubanga intervenue le 15 mars 2020 après avoir purgé une peine d'emprisonnement : « Comment peut-on

39 Le Procureur c. Thomas Lubanga Dyilo, Chambre de Première Instance II, Rectificatif de la « Décision fixant le montant des réparations auxquelles Thomas Lubanga Dyilo est tenu ", version publique expurgée, ICC-01/04-01/06 du 21 décembre 2017, ICC-01/04-01/06-3379-Red-Corr du 21 décembre 2017, paragraphes. 123/124. 
le libérer alors que la réparation n'est pas encore effective ? Pour Bosco [Ntaganda], nous pensons que la cour va le détenir jusqu'à la réparation des victimes car il est co-auteur de Thomas $"{ }^{40}$ s'est exclamée une des victimes ayant entendu parlé de la libération de Thomas Lubanga.

Visiblement, la situation est au point mort, la procédure est dans l'impasse et les victimes attendent toujours la pleine exécution de l'ordonnance de réparation (Redress Trust : 2016 , page $8 ; 2019,26)$. Depuis lors, le Fonds au Profit des victimes aura certainement de la peine à identifier et à localiser les victimes suite à la reprise des hostilités dans les zones cibles entre les groupes armés et les forces armées gouvernementales. A cela, il faudra aussi une politique bien ficelée pour qu'au moment venu, les opérations de réparations ne suscitent pas de nouvelles tensions entre les communautés locales directement concernées et ne soient pas aussi une nouvelle forme de traumatisme psychologique des victimes obligées aujourd'hui d'accueillir leur bourreau qui vaque librement à ses occupations ?

\section{LES RÉPARATIONS DANS L'AFFAIRE KATANGA}

La Chambre de première instance II a déclaré, en date du 7 mars 2014, Germain Katanga coupable, au sens de l'article 25-3-d du Statut, de complicité des crimes, commis le 24 février 2003, d'un chef de crime contre l'humanité (meurtre) et de quatre chefs de crime de guerre (meurtre, attaque contre une population civile en tant que telle ou contre des personnes civiles ne participant pas directement aux hostilités, destruction des biens et pillage) en lien avec l'attaque de Bogoro (Ituri/RDC).

Après une procédure fouillée, la Chambre de Première Instance II a rendu son ordonnance de réparation à l'encontre de $\mathrm{M}$. Germain Katanga en vertu de l'article 75 du Statut : ${ }^{41}$

«Constate que deux cent quatre-vingt-dix-sept des trois cent quarante et un Demandeurs ont démontré au standard de preuve de l'hypothèse la plus probable être victime des crimes commis pour lesquels $M$. Katanga a été coupable ;

Décide, par conséquent, que ces deux cent quatre-vingt-dix-sept victimes doivent bénéficier des réparations octroyées dans la présente affaire ;

Evalue l'ampleur du préjudice subi par les deux cent quatre-vingt-dix-sept victimes à une valeur monétaire totale de 3.752.620 USD ;

Fixe le montant incombant à $M$. Katanga en matière de réparations à 1.000.000 USD ;

Déclare que M. Katanga est indigent aux fins des réparations au jour de la présente Ordonnance de réparation ;

Ordonne des réparations individuelles, à savoir une indemnisation sous forme d'un montant symbolique de 250 USD ainsi que des réparations collectives ciblées au bénéfice de chaque victime, sous forme d'une aide au

40 Joachim Unegi, Directeur de la Radio Colombe de Mahagi (180 km au nord de Bunia, à la frontière avec l'Ouganda), Les victimes des crimes craignent pour l'effectivité de la réparation suite à la libération de Thomas Lubanga, 25 mars 2020, https://french.lubangatrial.org/2020/03/25/les-victimes-des-crimes-craignent-pour-leffectivite-de-la-reparation-suite-a-la-liberation-de-thomas-lubanga/

41 Le Procureur c. Germain Katanga, Chambre de Première Instance II, Ordonnance de réparation, ICC-01/04-01/07-3728 du 24 mars 2017. 
logement, d'un soutien à une activité génératrice de revenus, d'une aide à l'éducation et d'un soutien psychologique ;

Enjoint au Fonds de préparer un projet de mise en œuvre, à la lumière des décisions de la Chambre relatives aux types et modalités de réparations, qu'il déposera le 27 juin 2017 au plus tard et dans lequel il proposera un programme décrivant les projets qu'il entend développer ;

Enjoint au Représentant légal et à la Défense de déposer des observations sur le Projet pour le 28 juillet 2017 au plus tard ;

Enjoint à la Défense de contacter le Fonds afin de discuter de la contribution de M. Katanga, s'il le souhaite, aux modalités de réparations ;

Enjoint au Fonds de prendre contact avec le Gouvernement de la RDC sur sa possible collaboration à la réalisation et à la mise en œuvre des réparations ;

Enjoint à la Présidence, avec l'assistance du Greffier, de surveiller de manière continue la situation financière de $\mathrm{M}$. Katanga conformément à la norme 117 du Règlement de la Cour ;

Enjoint, au vu de la situation financière actuelle de M. Katanga, au Conseil de direction du Fonds de lui indiquer s'il est disposé à utiliser ses " autres ressources " afin de permettre le financement et la mise en œuvre des réparations individuelles et collectives, et de l'informer dudit montant dans le Projet ;

Invite le Fonds à tenir compte, dans le cadre de son mandat d'assistance, chaque fois que cela lui sera possible, des préjudices qu'ont subis les Demandeurs du fait des violences à caractère sexuel ou du fait d'un traumatisme psychique transgénérationnel ainsi que des préjudices qu'ont subis les anciens enfants soldats, que la Chambre n'a pas été en mesure de considérer dans la présente affaire ; et

Enjoint au Greffe de prendre toutes les mesures nécessaires pour donner une publicité adéquate à la présente Ordonnance de réparation ».

Par cette Ordonnance de réparation, la Chambre a fait preuve de quelques améliorations contrairement à l'Affaire Lubanga. ${ }^{42}$ Des centaines des victimes ont été identifiées par le Greffe et Représentant légal des victimes, des propositions concrètes sur l'évaluation monétaire du préjudice subi par les victimes ont été soumises à la Chambre. Toute la procédure a permis aux victimes de se manifester et de présenter leurs perspectives en spécifiant leur demande de réparation avant que celles-ci ne soient octroyées (Redress trust : 2016, page 9). ${ }^{43}$ Cela, pour éviter toute indifférence de la part de victimes et des tensions entre communautés, les réparations doivent s'inspirer de la culture et des coutumes locales qui ne sont pas discriminatoires ou d'exclusion. ${ }^{44}$ L'affaire Katanga rencontre tous nos suffrages par le fait que la Cour, « pour la première fois, a accordé des réparations à des victimes individuelles

42 L'Affaire Lubanga a connu trois ordonnances de réparation dont la dernière est : Le Procureur c. Thomas Lubanga Dyilo, Chambre de Première Instance II, Rectificatif de la « Décision fixant le montant des réparations auxquelles Thomas Lubanga Dyilo est tenu ", version publique expurgée, ICC-01/04-01/06 du 21 décembre 2017, ICC-01/04-01/06-3379-Red-Corr du 21 décembre 2017. Les améliorations sont notamment la qualité de l'ordonnance sous examen et d'autres aspects comme les réparations individuelles accordées tout en s'inspirant de la culture et des coutumes locales non discriminatoires des victimes.

43 CPI, Katanga, ICC-01/04-01/07-3711, 30 septembre 2016, Observations de la Défense sur l'évaluation monétaire du préjudice subi par les victimes ; CPI, Katanga, ICC-01/04-01/07-3713, 30 septembre 2016, Observations des victimes sur la valeur monétaire des préjudices allégués. 01/06-3129-AnxA-tFRA, le 1er août 2016, paragraphe 47. 
... Elles ont ainsi, à la fin de la procédure, obtenu une indemnisation financière symbolique en plus d'une aide au logement et d'un soutien à une activité génératrice de revenus, ainsi que des réparations collectives" (Redress Trust : 2016, page 9 ; 2019 : page 27). Visiblement, ces réparations ont aussi été orienté vers des programmes autonomes en vue de permettre aux victimes, à leurs familles et à leurs communautés de bénéficier de ces réparations sur le long terme. ${ }^{45}$ Les premières actions individuelles faites par la CPI à Bogoro ${ }^{46}$ méritent de faire l'objet d'une évaluation soutenue tant du côté de la Cour que des victimes bénéficiaires sur la valeur des montants alloués individuellement par rapport au pouvoir d'achat de la population, du contexte sécuritaire, de la réparation individuelle directe et de la réparation individuelle indirecte.

Tout en rappelant les principes établis en matière de réparation (article 75-1 du Statut), en appréciant les efforts de la Chambre d'appel dans l'affaire Lubanga et sous réserve des modifications contenues dans l'Ordonnance de réparation dans l'affaire Katanga (Ordonnance de réparation sous examen ici), la Chambre rappelle en particulier que :

«Pour toutes les questions liées aux réparations, elle « ... doit traiter les victimes avec humanité et respecter leur dignité et leurs droits humains ». La Chambre doit également traiter toutes les victimes ... équitablement et de la même manière, qu'elles aient participé ou non au procès ayant débouché sur la décision rendue en application de l'article 74 du Statut ... La Chambre rappelle en outre que, tel qu'énoncé à l'article 68 du Statut et à la règle 86 du Règlement de Procédure et de Preuve, elle doit tenir compte des besoins de toutes les victimes. La Chambre rappelle également que, conformément aux règles 87 et 88 du Règlement de Procédure et de Preuve, des mesures appropriées doivent être mises en œuvre afin de garantir la sécurité, le bien-être physique et psychologique et la protection de la vie privée des victimes. Il est, par ailleurs, primordial que les réparations soient accordées et accessibles aux victimes sans distinction défavorable fondée sur le sexe, l'âge, la race, la couleur, la langue, la religion ou la conviction, les opinions politiques ou autres, I'orientation sexuelle, I'origine nationale, ethnique ou sociale, la fortune, la naissance ou toute autre qualité $» .{ }^{47}$

La Chambre a aussi tenu à rappeler qu'une ordonnance de réparation rendue en vertu de l'article 75 du Statut doit répondre, au minimum, à cinq critères essentiels :

- I'ordonnance de réparation doit être rendue à l'encontre de la personne déclarée coupable ;

- la Chambre doit indiquer quelles sont les victimes admises à bénéficier des réparations accordées ou fixer les critères d'admissibilité sur base du lien entre le préjudice subi par les victimes et les crimes dont la personne a été déclarée coupable ;

- la Chambre doit définir le préjudice causé aux victimes du fait des crimes dont la personne a été déclarée coupable. À cet égard, la Chambre note que l'évaluation de l'ampleur du préjudice causé aux victimes, aux fins de définir la nature et/ou l'importance des réparations à octroyer, peut

45 Le Procureur c. Thomas Lubanga Dyilo, Chambre d'appel, Ordonnance de réparation modifiée, du 3 mars 2015, ICC-01/0401/06-3129-AnxA-tFRA, le 1er août 2016, paragraphe 48.

46 Fonds au profit des victimes, Assistance et réparations, réalisations, enseignements tirés et évolution, Rapport sur l'état d'avancement des programmes, Septembre 2015, Page 65.

47 Lubanga, Chambre d'appel, Ordonnance de réparation, ICC-01/04-01/06-3129-AnxA-tFRA, paragraphes 15, 12, 34, 18 et 16; Le Procureur c. Germain Katanga, Chambre de Première Instance II, Ordonnance de réparation, ICC-01/04-01/07-3728 du 24 mars 2017, paragraphe 30 . 
être effectuée par une Chambre de première instance, dans l'ordonnance de réparation, ou être effectuée par le Fonds une fois l'ordonnance de réparation rendue ;

- la Chambre doit établir la responsabilité de la personne coupable en matière de réparations et l'informer de cette responsabilité. Cela signifie que la chambre doit préciser la portée de cette responsabilité en fixant le montant monétaire qui lui incombe à ce titre. A cet égard, la Chambre note que la responsabilité en matière de réparations d'une personne déclarée coupable se fonde sur et est limitée aux préjudices causés par les crimes pour lesquels la personne a été reconnue coupable ...

- la Chambre doit préciser et motiver le type de réparations ordonnées, qu'elles soient collectives, individuelles ou les deux, conformément aux règles 97-1 et 98 du Règlement de procédure et de preuve. Elle doit aussi indiquer les modalités de réparations que la Chambre juge appropriées sur la base des circonstances particulières de l'affaire en l'espèce. ${ }^{48}$

Rappelant aussi la notion de « victime » au sens de l'article 85 du Règlement de Procédure et de Preuve, la Chambre est revenue sur quatre conditions présentée par la Chambre d'appel dans l'affaire Lubanga ${ }^{49}$ avant d'accorder le statut de victime participant au stade du procès pour toute personne ayant présenté une demande de participant, à savoir : « le demandeur doit être une personne physique ou morale ; doit avoir subi un préjudice ; que le crime ayant causé préjudice relève de la compétence de la Cour et qu'il existe un lien de causalité entre ledit préjudice et le crime. La notion de victime implique nécessairement l'existence d'un préjudice personnel, mais aussi n'implique pas nécessairement l'existence d'un préjudice direct $» .^{50}$

La libération de Germain Katanga avant l'effectivité de réparations des victimes en Ituri soulève aujourd'hui beaucoup d'inquiétudes dans les milieux des victimes. Elles sont très désemparées quant à leur sort tant sur le plan psychologique que sécuritaire. Comment garantir les droits des victimes conformément aux prescrits des articles 87 et 88 du Règlement de Procédure et de Preuve après la libération du coupable ayant purgé sa peine ? Est-ce que la Cour a communiqué des mesures appropriées afin de garantir la sécurité, le bien-être physique et psychologique et la protection de la vie privée des victimes ? Quelle appréciation faire de la lenteur qui caractérise le processus d'indemnisation des victimes ? Toutes considérations faites, et si aucune action n'est entreprise dans le meilleur délai, d'aucuns seraient tenté à voir le travail de la Cour sous un œil d'une " pièce de cinéma » sans impact réel sur le sort des victimes en termes de réparation et de garantie de non répétition particulièrement.

\section{LES RÉPARATIONS DANS L'AFFAIRE AL MAHDI}

En date du 27 septembre 2016, Ahmad Al Faqi Al Mahdi a été reconnu par la CPI coupable du crime de guerre d'avoir intentionnellement dirigé des attaques contre des monuments

48 Le Procureur c. Germain Katanga, Chambre de Première Instance II, Ordonnance de réparation, ICC-01/04-01/07-3728 du 24 mars 2017, paragraphe 31.

49 Le Procureur c. Thomas Lubanga Dyilo, Chambre d'appel, Arrêt relatif aux appels interjetés par le Procureur et la Défense contre la Décision relative à la participation des victimes rendues le 18 janvier 2008 par la Chambre de première instance I, daté le 11 juillet 2008 et traduction enregistrée le 27 août 2008, ICC-01/04-01/06-1432-tFRA, paragraphes 61-65. 
historiques et dédiés à la religion à Tombouctou, au Mali, entre fin juin et début juillet en 2012. ${ }^{51}$ Dans son Ordonnance de réparation, la Chambre de première instance VIII :

"Ordonne l'octroi de réparations individuelles, collectives et symboliques en faveur de la communauté de Tombouctou, comme précisé aux paragraphes $56,67,71,83,90,106$ et 107 de la présente ordonnance,

Reconnaît que la destruction des Bâtiments protégés a causé des souffrances à toute la population du Mali et à la communauté internationale,

Evalue la responsabilité d'Ahmad Al Mahdi aux fins de ces réparations à 2,7 millions d'euros,

Encourage le Fonds au profit des victimes à prendre des mesures pour compléter les réparations ordonnées et à proposer aux victimes au Mali une assistance plus large, comme précisé aux paragraphes 108 et 138 de la présente ordonnance,

Ordonne au Greffe de prendre immédiatement les mesures symboliques définies au paragraphe 71 de la présente ordonnance,

Fixe au 16 février 2018 la date limite de dépôt du projet de plan de mise en œuvre préparé par le Fonds au profit des victimes, et

Donne instruction au représentant légal des victimes et à la Défense de déposer toute observation concernant le projet de plan de mise en œuvre dans un délai de 30 jours à compter de sa notification $» .52$

A l'instar des affaires Lubanga et Katanga, la Chambre reconnaît l'état d'indigence d'Ahmad Al Mahdi tout en encourageant le Fonds au profit des victimes à compléter les mesures de réparation individuelles et collectives et à s'employer à collecter des fonds dans la mesure nécessaire pour compléter la totalité des mesures ordonnées. ${ }^{53}$

Par son rapport, The Redress Trust (2016, page 11), estime que l'affaire Ahmad Al Faqi Al Mahdi (Al Mahdi) se présente en un modèle d'efficacité et de rapidité (bien qu'il faille tenir compte de la circonstance particulière que constitue l'admission de culpabilité). II a fallu seulement un an à la Cour pour conduire les procédures préliminaires et le procès après la présentation de Ahmad Al Faqi Al Mahdi devant elle, et produire une Ordonnance de réparation le 17 août 2017. En revenant sur la question des victimes dans cette affaire, la Chambre « reconnaît que la destruction des Bâtiments protégés a causé des souffrances à toute la population du Mali et à la communauté internationale ». Les victimes ne sont pas seulement Maliennes mais aussi toute la communauté internationale, ce qui fait que le nombre des victimes devient plus important et nécessitant des réparations significatives. L'indigence du coupable ne constitue pas un obstacle à la réparation des victimes. Ainsi, la Chambre a alloué des réparations individuelles, collectives et symboliques aux victimes. Il a aussi été considéré que les descendants des personnes dont les membres de la famille avaient été enterrés dans les mausolées endommagés pouvaient recevoir une indemnisation pour le préjudice mental. Pour le fait que la destruction des sites avait engendré de l'angoisse et des souffrances morales chez les victimes individuelles et la communauté de Tombouctou, une compensation symbolique de 1 euro a été octroyé au Mali et à l'Unesco pour les dommages subis

51 Le Procureur c. Ahmad Al Faqi Mahdi, ICC-01/12-01/15-171-tFRA, 2 Septembre 2016.

52 Le Procureur c. Ahmad Al Faqi Al Mahdi, Ordonnance de réparation, ICC-01/12-01/15-236-tFRA du 17 août 2017, VII, paragraphes 66/67.

53 Ibidem, paragraphe 138 
par le Mali et la communauté internationale. II s'agit ici d'une avancée aussi importante de reconnaissance et de réparations des torts causés au Mali et à la communauté internationale (Redress Trust : 2019, page 27), car les biens inscrits sur la liste du patrimoine commun de l'humanité bénéficient d'une protection à juste titre.

\section{LES RÉPARATIONS DANS LES AFFAIRES NGUDJOLO, BEMBA ET RUTO}

Par le jugement d'acquittement du 18 décembre 2012 et confirmé par la Chambre d'appel, le 7 avril 2015, aucune ordonnance de réparation n'a été rendue dans l'affaire Ngudjolo. Tel est aussi le cas des affaires The Prosecutor v. William Samoei Ruto and Joshua Arap Sang ${ }^{54}$ dans laquelle le juge avait décidé de mettre fin au procès suite à l'insuffisance des moyens à charge ; et Le Procureur c. Jean Pierre Bemba, ${ }^{55}$ où la Chambre d'appel a considéré que les crimes visés au paragraphe 116 du jugement attaqué (jugement de condamnation) n'entraient pas dans le cadre des faits et circonstances décrits dans les charges. Par voie de conséquence elle a procédé à l'annulation du jugement attaqué et acquitté Jean Pierre Bemba de toutes les autres charges contre lui.

« ... aucune ordonnance de réparation ne peut être rendue à l'encontre de Jean Pierre Bemba en vertu de l'article 75 du Statut. La Chambre se doit de respecter les limites de la Cour et rappelle qu'elle ne peut ordonner l'octroi de réparations pour le préjudice subi du fait de crimes que si la personne jugée pour sa participation à ces crimes a été déclarée coupable. Toutefois, la Cour a été créée pour remplir une fonction aussi bien punitive que réparatrice (article 68-3 du Statut), ... il relève de son pouvoir de rendre une décision finale relative à la procédure en réparation, ayant elle-même mené l'ensemble des procédures en première instance et en réparation dans cette affaire. Elle considère qu'il convient de prendre acte des vues et préoccupations des victimes, conformément à l'article 68-3 du Statut, et juge que la Décision finale n'est ni préjudiciable ni contraire aux droits de Jean Pierre Bemba ». ${ }^{56}$

Contrairement aux autres affaires devant la CPI, le cas Jean Pierre Bemba est un cas d'école lorsqu'on se permet de s'intéresser au sort des victimes. Tout au long de la procédure en premier degré avant de délivrer une "ordonnance relative à la réparation ", plus de 5.000 victimes avaient participé au procès Bemba, une quantité importante des biens/fonds appartenant à Mr Bemba venaient d'être identifiés et gelés. Il était donc hors de question d'envisager une situation d'indigence dans la situation Bemba comme cela était les cas respectivement dans les affaires Lubanga et Katanga. Considérant le sort du prévenu condamné en 1er ressort et acquitté en appel, Redress Trust (2019, page $27 ; 2016$, Page 11) a soulevé une préoccupation fondamentale dans son rapport : Est-il prudent pour la Cour de commencer à auditionner les parties concernant les réparations avant d'avoir déterminé les dernières questions d'appel ? Les résultats des consultations réalisées par Redress Trust ( 2019, page 65) révèlent que

54 The Prosecutor v. William Samoei Ruto and Joshua Arap Sang, trial Chamber V (A), Decision of Defense Applications for Judgment of Acquittal, ICC-01/09-01/11, 5 april 2016, ICC-01/09-01/11-2027-Red-Corr du 16 june 2016, P. 1, paragraphe 149 ; Le Procureur c. Jean Pierre Bemba, Chambre d'Appel, ICC-01/05-01/08-3653-tFRA du 3 août 2018, paragraphe 3.

55 Le Procureur c. Jean Pierre Bemba, Chambre d'Appel, ICC-01/05-01/08 A, 8 Juin 2018, ICC-01/05-01/08-3636-Red-tFRA du 16 juillet 2018, page 5 .

56 Le Procureur c. Jean Pierre Bemba, Chambre d'Appel, ICC-01/05-01/08-3653-tFRA du 3 août 2018, paragraphe 3. 
«Les réparations peuvent et devraient être intégrées à la procédure au stade préliminaire et en première instance. Les parties et participants seraient en mesure de formuler des observations plus ciblées, et le Greffe et le Fonds au profit des victime pourraient fournir plus d'informations utiles sur les détails pratiques de l'affaire particulière en question. Et surtout, de tels principes donneraient aux victimes une idée de ce à quoi elles peuvent s'attendre, que ce soit sur le plan procédural et sur le plan fond ».

Tout en saluant le souci de protection de victimes au cours de toute la chaine de la procédure pénale, il n'est pas aussi indiqué d'ignorer la pertinence de respect du droit de l'accusé à un procès juste et équitable. La décision d'acquittement de la Chambre d'appel dans l'affaire Bemba éclaire notre religion quant au besoin de protection et de respect des droits de toutes les parties au procès et le sort des victimes reconnues en faisant appel à la mise en œuvre du deuxième mandat du Fonds au profit des victimes (mandat d'assistance). Le représentant légal des victimes a entre autres comme devoir d'informer les victimes sur la procédure en réparation qui est à la fois liée à la procédure pénale et séparée de cette dernière pour ne pas alimenter toute spéculation inconsidérée.

Le caractère sui generis de l'affaire Bemba porte particulièrement ici sur le fait que les victimes ont existé mais le coupable des crimes ayant causé des préjudices n'existe pas. Ainsi, la Chambre n'a pas hésité à reconnaître les victimes en ces termes :

«... la décision rendue par la Chambre d'appel ne reposait sur un quelconque doute quant au préjudice subi par les victimes ayant participé à la procédure ... La Chambre d'appel a reconnu que certains crimes ont eu lieu en RCA entre 2002 et 2003 et n'a donc pas remis en question le statut des victimes en tant que tel ... La Chambre reconnaît que d'autres personnes, qui n'ont pas été admises à participer en tant que victimes en l'espèce, ont pu subir un préjudice du fait des crimes relevant de la compétence de la Cour en RCA entre 2002 et 2003 et devraient donc également être considérées comme des victimes aux fins du mandat d'assistance du Fonds. Au vu du nombre élevé de victimes en l'espèce et de la situation difficile en matière de sécurité en RCA, la mise en œuvre par le Fonds d'un programme relevant de son mandat d'assistance sera sans doute une tâche délicate. La réussite de tout programme dépendra largement de la capacité du Fonds d'obtenir, entre autres, des données à jour sur les victimes, comme les renseignements leur permettant de les joindre et le lieu où elles se trouvent, et de bénéficier des réseaux de partenaires sur place. Étant donné que bon nombre de ces informations ont déjà été recueillies tout au long de la procédure en réparation par les représentants légaux et les sections concernées du Greffe, la Chambre encourage fortement toutes les parties prenantes à coopérer avec le Fonds ${ }^{.57}$

Le Fonds au profit des victimes devrait s'engager dans l'exécution de son deuxième mandat d'apporter assistance aux victimes en République Centrafricaine (RCA). Les victimes ont non seulement crié à l'impunité entretenue par la Cour en RCA, selon elles, en perdant ainsi confiance en la justice de la Cour après l'acquittement de Jean Pierre Bemba. ${ }^{58}$ Une telle lecture serait réductrice de la mission de la Cour appelée à rendre une justice juste et équitable en respectant les droits de toutes les parties au procès. La reconnaissance des

57 Le Procureur c. Jean Pierre Bemba, Décision finale relative à la procédure en réparation, ICC-01/05-01/08 du 3 août 2018, ICC-01/05-01/08-3653-tFRA du 3 août 2018 paragraphes 6, 12.

58 Ibidem, paragraphe 6 . 
victimes a été une manifestation correcte de la réelle volonté de la Cour à ne pas abandonner les victimes à leur triste sort, le Fonds au profit des victimes dans son mandat d'assistance apparaissant ici comme un mécanisme solide de leur prise en charge.

La phase de la réparation est marquée par des entraves de plusieurs ordres qui ne permettent pas une mise en œuvre effective des réparations dans le meilleur délai. Cet état des choses est aussi critiquable au point que « pour les victimes, ces longues et pénibles années d'attente pour obtenir justice ont été une succession d'espoirs et de déceptions, de craintes et de joies. " (Redress Trust : 2019, page 9). Une simple impression conduirait à penser que la Cour a été " chaussée " par les Etats parties au Statut de Rome au-delà de sa taille, n'ayant pas tous les moyens de sa politique en matière de réparation, elle court le risque d'un déshonneur ou d'une considération de "théâtralisation » aux yeux des victimes. Au-delà du cliché financier, elle rencontre des difficultés énormes à conjuguer aisément avec le Fonds au profit des victimes spécifiquement sur la question de plan de mise en œuvre de la réparation. A cela, il faut ajouter toute l'impasse autour du moment de la formulation et de l'introduction d'une demande en réparation, l'identification des victimes potentielles bénéficiaires de la réparation, l'évaluation des demandes déclarées recevables par la Cour, l'évaluation du préjudice subi et l'établissement de la responsabilité civile du coupable, le manque d'homogénéité dans les décisions rendues, l'absence de stratégies en matière de réparations à l'échelle de la Cour (Redress Trust : 2019, page 11-14;2016, pages 11 -22). Considérant le caractère aussi complexe de la procédure en matière de réparation qui peut conduire au risque d'exclure certaines victimes, et, susciter par ce fait, des nouvelles tensions entre les communautés locales, la Cour devra renforcer son programme de sensibilisation auprès des victimes.

\section{CONCLUSION}

Le droit à la réparation en matière des crimes internationaux est aujourd'hui consacré en droit international pénal par le système du Statut de Rome de la CPI et par d'autres instruments juridiques internationaux à titre d'un droit fondamental. Ce qui n'avait jamais été accordé devant une juridiction pénale internationale est désormais une réalité devant la $\mathrm{CPI}$. Les victimes des crimes relevant de sa compétence jouissent de leur droit à la réparation. Cette évolution permet aux victimes de partager des informations au Procureur de la Cour et lui demander d'ouvrir des enquêtes, de témoigner au cours d'un procès, de participer aux procédures en exprimant leurs vues et préoccupations aux juges et formuler des demandes de réparation du préjudice qu'elles ont subi. La participation à la procédure n'est cependant pas un préalable pour avoir droit à la réparation. Une victime qui n'a pas participé à la procédure, peut très bien faire une demande de réparation. La Cour peut même accorder une réparation d'office. II est envisagé un mécanisme approprié mettant les victimes à l'abris de l'insolvabilité ou de l'indigence du coupable, ou alors, en cas d'acquittement du prévenu après avoir reconnu l'existence des victimes. Le Fonds au profit des victimes est l'institution établie en vue de procéder aux réparations conformément aux ordonnances rendues par la Cour ou alors d'assister les victimes reconnues. 
Le Fonds au profit des victimes, un mécanisme du système des réparations, a été sollicité en réparation comme en assistance aux victimes dans les différentes ordonnances de réparation prononcées par la Cour. II a été fait recours notamment à des compensations monétaires, de restitution des biens, des mesures de réhabilitation, des mesures symboliques telles que des excuses ou des commémorations. II a été accordé soit une réparation individuelle, soit une réparation collective, soit les deux concurremment selon ce qui convient le mieux aux victimes dans l'affaire considérée.

Dix-huit ans après la création de la Cour et près de huit ans après la publication de la première ordonnance de réparation en 2012 (Affaire Lubanga), la Cour vient de réaliser des avancées notables en termes de consolidation d'une jurisprudence qui fait école en matière des réparations. Néanmoins les résultats des réparations (affaires Lubanga, Katanga en RDC et Al Mahdi au Mali) et d'assistance aux victimes (affaire Bemba en République Centrafricaine) sont très mitigés. L'exécution effective et rapide des réparations en faveur des victimes est indûment retardée (Redress Trust : 2019, page 14). La procédure pénale semble l'avoir emporté sur la procédure en réparation au point que les victimes s'interrogent sur la ratio legis de toutes les ordonnances de réparation rendues. La mise en œuvre de ces ordonnances n'est plus à négocier, il s'agit d'un droit reconnu aux victimes. Visiblement, les ordonnances n'ont pas franchi le seuil du prétoire, elles demeurent des simples chapelets d'intentions sans impact réel sur les victimes dans leurs milieux de vie. Le retard observé dans la réalisation effective des réparations (droit à la réparation) devra attirer l'attention des représentants légaux des victimes et du Bureau du conseil public pour les victimes (BCPV). Le mandat de représentation et d'assistance juridique aux victimes confié aux avocats et au BCPV n'arrive à terme qu'après une réparation effective du préjudice causé aux victimes et l'assistance à accorder. Toute manœuvre dilatoire doit être dénoncée au plus haut niveau devant les instances habilitées. Le dépassement excessif de délai serait compromettant au point que les victimes seraient encore exposées au châtiment de leurs anciens bourreaux qui reviennent après avoir purgé leurs peines sans que des mesures de sécurité des victimes n'aient été envisagées ni par la Cour ou ni par l'Etat. La libération de Germain Katanga et de Thomas Lubanga et leur éventuel retour en Ituri en RDC avant que les réparations ne soient effectives alimente un sentiment d'abandon de victimes au profit des bourreaux.

L'espoir des milliers des victimes en RDC comme en RCA à la justice réparatrice annoncée " tambour battant " à Rome, les victimes ne pouvant désormais obtenir réparation, semble être une coquille vide. Le retour à la case de départ quant au sort des victimes devant les juridictions pénales internationales semble redevenir une réalité, car « chassez le naturel, il revient au galop ». Le succès de la Cour tient, certes, non seulement au fait de condamner les criminels, mais aussi, à sa capacité à mettre en place un système de réparations solide dans sa manière de rendre la justice. Le renforcement des unités de sensibilisation dans les pays membres, particulièrement où on enregistre des situations et des affaires pendantes devant la Cour s'avère une nécessité. Tout en fustigeant les faiblesses et le retard observés dans la procédure des réparations devant la CPI, Redress Trust (2019, pages 15-16) présente un menu des recommandations à mettre à profit. Les représentants légaux des victimes (avocats) doivent jouer en amont comme en aval un rôle proactif veillant à la réalisation effective de la réparation dans un "délai raisonnable ", dénoncer les obstacles éventuels et garantir leur protection lors de la libération des bourreaux. La Cour a encore un long chemin à parcourir. Elle doit relever le défi de dépasser le simple slogan et la volonté théorique de prise 
en compte de besoins des victimes (droit à la réparation) et atteindre la phase effective des réparations des victimes ayant subi des préjudices des crimes relevant de sa compétence. Le tableau ci-dessous ${ }^{59}$ est un miroir ou une vitrine jurisprudentielle de la Cour (jurisprudence) dans sa phase de mise en œuvre des réparations au profit des victimes des crimes internationaux relevant de sa compétence.

\begin{tabular}{|c|c|c|c|c|c|}
\hline $\mathrm{N}^{\circ}$ & Affaires & Décisions & $\begin{array}{l}\text { Niveau d'exécution : } \\
\text { Total/partiel-raison }\end{array}$ & $\begin{array}{l}\text { Lieu } \\
\text { d'exécu- } \\
\text { tion }\end{array}$ & $\begin{array}{c}\text { Observations/commen- } \\
\text { taires }\end{array}$ \\
\hline 01. & $\begin{array}{l}\text { Le Procureur } \\
\text { C. Mathieu } \\
\text { Ngudjolo }\end{array}$ & $\begin{array}{l}\text { - Jugement d'ac- } \\
\text { quittement } \\
\text { - Pas d'ordonnance } \\
\text { de réparation, re- } \\
\text { cours au mandat } \\
\text { d'assistance du } \\
\text { Fonds au Profit } \\
\text { des victimes }\end{array}$ & $\begin{array}{l}\text { Aucune ordonnance de } \\
\text { réparation n'ayant été } \\
\text { prononcée, aucune répa- } \\
\text { ration n'est en cours. }\end{array}$ & Ituri/RDC & $\begin{array}{l}\text { Le mandat d'assistan- } \\
\text { ce du Fonds s'étend à } \\
\text { toutes les victimes des } \\
\text { crimes tombant sous la } \\
\text { compétence de la Cour, } \\
\text { sans considération de } \\
\text { leur auteur. } \\
\text { Le recours au mandat } \\
\text { d'assistance du Fonds } \\
\text { n'est pas alternatif à } \\
\text { l'absence de réparations } \\
\text { accordées dans une } \\
\text { affaire. Il s'exécute indé- } \\
\text { pendamment des procé- } \\
\text { dures de réparation. }\end{array}$ \\
\hline 02. & $\begin{array}{l}\text { Le Procureur } \\
\text { C. Thomas } \\
\text { Lubanga } \\
\text { Dyilo }\end{array}$ & $\begin{array}{l}\text { - Jugement de } \\
\text { condamnation } \\
\text { - (Indigent) } \\
\text { - Ordonnance de } \\
\text { réparation dispo- } \\
\text { nible }\end{array}$ & $\begin{array}{l}\text { L'ordonnance de répara- } \\
\text { tion est en cours d'exé- } \\
\text { cution. } \\
\text { Le Fonds au profit des } \\
\text { victimes a présenté un } \\
\text { projet de plan de mise en } \\
\text { œuvre de l'ordonnance } \\
\text { de réparation et diverses } \\
\text { informations addition- } \\
\text { nelles. La Chambre de } \\
\text { première instance com- } \\
\text { pétente a approuvé la } \\
\text { mise en œuvre de répa- } \\
\text { rations collectives sym- } \\
\text { boliques ainsi que des } \\
\text { réparations collectives } \\
\text { sous forme de services } \\
\text { apportés aux victimes. } \\
\text { Le Fonds a entamé les } \\
\text { démarches administra- } \\
\text { tives nécessaires à la } \\
\text { mise en œuvre concrète } \\
\text { de ces activités. } \\
\text { L'identification des vic- } \\
\text { times est bien avancée. } \\
\text { Aucune information pu- } \\
\text { blique supplémentaire ne } \\
\text { permet d'établir le niveau } \\
\text { d'exécution des répara- } \\
\text { tions avec précision. }\end{array}$ & Ituri/RDC & $\begin{array}{l}\text { Les informations sont } \\
\text { confidentielles afin } \\
\text { d'assurer le succès de } \\
\text { la mise en œuvre et } \\
\text { garantir la sécurité des } \\
\text { victimes concernées } \\
\text { qui sont informées du } \\
\text { niveau d'exécution. }\end{array}$ \\
\hline
\end{tabular}




\begin{tabular}{|c|c|c|c|c|c|}
\hline 03. & $\begin{array}{l}\text { Le Procureur } \\
\text { c. Germain } \\
\text { Katanga }\end{array}$ & $\begin{array}{l}\text { - Jugement de } \\
\text { condamnation } \\
\text { - (Indigent) } \\
\text { - Ordonnance de } \\
\text { réparation dispo- } \\
\text { nible }\end{array}$ & $\begin{array}{l}\text { L'ordonnance de répara- } \\
\text { tion est en cours d'exé- } \\
\text { cution. } \\
\text { La Chambre a accordé } \\
\text { des réparations indi- } \\
\text { viduelles symboliques } \\
\text { ainsi que des réparations } \\
\text { collectives ciblées aux } \\
\text { individus qu'elle a recon- } \\
\text { nu comme victimes. } \\
\text { Le Fonds au profit des } \\
\text { victimes a présenté un } \\
\text { projet de plan de mise en } \\
\text { œuvre de l'ordonnance } \\
\text { de réparation et diverses } \\
\text { informations addition- } \\
\text { nelles. } \\
\text { Le Fonds a entamé les } \\
\text { démarches administra- } \\
\text { tives nécessaires à la } \\
\text { mise en œuvre concrète } \\
\text { de ces activités. } \\
\text { Aucune information pu- } \\
\text { blique supplémentaire ne } \\
\text { permet d'établir le niveau } \\
\text { d'exécution des répara- } \\
\text { tions avec précision. }\end{array}$ & Ituri/RDC & $\begin{array}{l}\text { Les informations sont } \\
\text { confidentielles afin } \\
\text { d'assurer le succès de } \\
\text { la mise en œuvre et } \\
\text { garantir la sécurité des } \\
\text { victimes concernées } \\
\text { qui sont informées du } \\
\text { niveau d'exécution. }\end{array}$ \\
\hline 04. & $\begin{array}{l}\text { Le Procureur } \\
\text { c. JP Bemba }\end{array}$ & $\begin{array}{l}\text { - Jugement d'ac- } \\
\text { quittement } \\
\text { - Pas d'ordonnance } \\
\text { de réparation, re- } \\
\text { cours au mandat } \\
\text { d'assistance du } \\
\text { Fonds au Profit } \\
\text { des victimes }\end{array}$ & $\begin{array}{l}\text { Aucune ordonnance de } \\
\text { réparation n'ayant été } \\
\text { prononcée, aucune répa- } \\
\text { ration n'est en cours. }\end{array}$ & $\mathrm{RCA}$ & $\begin{array}{l}\text { Le mandat d'assistan- } \\
\text { ce du Fonds s' étend à } \\
\text { toutes les victimes de } \\
\text { crimes tombant sous la } \\
\text { compétence de la Cour, } \\
\text { sans considération de } \\
\text { leur auteur. } \\
\text { Le recours au mandat } \\
\text { d'assistance du Fonds } \\
\text { n'est pas alternatif à } \\
\text { l'absence de réparations } \\
\text { accordées dans une } \\
\text { affaire. Il s'exécute indé- } \\
\text { pendamment des procé- } \\
\text { dures de réparation. } \\
\text { Le Fonds finalise les } \\
\text { préparatifs pour la mise } \\
\text { en œuvre d'activités } \\
\text { spécifiques au profit } \\
\text { des victimes de crimes } \\
\text { tombant sous la compé- } \\
\text { tence de la Cour en RCA. }\end{array}$ \\
\hline
\end{tabular}




\begin{tabular}{|c|c|c|c|c|c|}
\hline 05. & $\begin{array}{l}\text { Le Procureur } \\
\text { c. } \\
\text { Ahmad Al } \\
\text { Faqi Al Mahdi }\end{array}$ & $\begin{array}{l}\text { - Jugement de } \\
\text { condamnation } \\
\text { - Ordonnance de } \\
\text { réparation dispo- } \\
\text { nible }\end{array}$ & $\begin{array}{l}\text { L'ordonnance de répara- } \\
\text { tion est en cours d'exé- } \\
\text { cution. } \\
\text { Le Fonds au profit des } \\
\text { victimes a présenté un } \\
\text { projet de plan de mise en } \\
\text { œuvre de l'ordonnance } \\
\text { de réparation et diverses } \\
\text { informations addition- } \\
\text { nelles. La Chambre de } \\
\text { première instance com- } \\
\text { pétente a approuvé la } \\
\text { mise en œuvre de répa- } \\
\text { rations individuelles et } \\
\text { collectives. } \\
\text { L'identification des vic- } \\
\text { times est bien avancée. } \\
\text { Le Fonds a entamé les } \\
\text { démarches administra- } \\
\text { tives nécessaires à la } \\
\text { mise en œuvre concrète } \\
\text { de ces activités. } \\
\text { Aucune information pu- } \\
\text { blique supplémentaire ne } \\
\text { permet d'établir le niveau } \\
\text { d'exécution des répara- } \\
\text { tions avec précision. }\end{array}$ & Mali & $\begin{array}{l}\text { Les informations sont } \\
\text { confidentielles afin } \\
\text { d'assurer le succès de } \\
\text { la mise en œuvre et } \\
\text { garantir la sécurité des } \\
\text { victimes concernées } \\
\text { qui sont informées du } \\
\text { niveau d'exécution. }\end{array}$ \\
\hline 06. & $\begin{array}{l}\text { The Pros- } \\
\text { ecutor c. Wil- } \\
\text { liam Samoei } \\
\text { Ruto and } \\
\text { Joshua Arap } \\
\text { Sang }\end{array}$ & $\begin{array}{l}\text { - Pas de jugement, } \\
\text { les juges ayant mis } \\
\text { fin au procès }\end{array}$ & $\begin{array}{l}\text { Aucune ordonnance de } \\
\text { réparation n'ayant été } \\
\text { prononcée, aucune répa- } \\
\text { ration n'est en cours. }\end{array}$ & Kenya & $\begin{array}{l}\text { Le mandat d'assistan- } \\
\text { ce du Fonds s' étend à } \\
\text { toutes les victimes de } \\
\text { crimes tombant sous la } \\
\text { compétence de la Cour, } \\
\text { sans considération de } \\
\text { leur auteur. } \\
\text { Le recours au mandat } \\
\text { d'assistance du Fonds } \\
\text { n'est pas alternatif à } \\
\text { l'absence de réparations } \\
\text { accordées dans une } \\
\text { affaire. Il s'exécute indé- } \\
\text { pendamment des procé- } \\
\text { dures de réparation. } \\
\text { Le Fonds a indiqué son } \\
\text { intention de mettre en } \\
\text { œuvre des activités } \\
\text { spécifiques au profit } \\
\text { des victimes de crimes } \\
\text { tombant sous la com- } \\
\text { pétence de la Cour au } \\
\text { Kenya. }\end{array}$ \\
\hline
\end{tabular}

\section{RÉFÉRENCES}

Bakama Bope E. \& Ingange wa Ingange J.D. (s/dir), Recueil des cours intensifs sur les droits de l'homme et le droit international pénal, Editions CAD, Kinshasa, 2015.

Bititi Gilbert, «Les victimes devant la Cour Pénale Internationale : Les promesses de Rome ont-elles été tenues ?» in Revue de Sciences Criminelles, 2011. 
Boré J., L'indemnisation pour les chances perdues : une forme d'appréciation quantitative de la causalité d'un fait dommageable, JCP, 1974.

Chabas F., L'influence de la pluralité des causes sur le droit à réparation, Thèse de doctorat, Paris, 1965.

Chan J., Le Fonds au profit des victimes de la Cour pénale internationale, Bruxelles, 26 Novembre 2013.

Christian Assale, Le droit du dommage corporel, système d'indemnisation, Dalloz, 1990.

Cour Pénale Internationale, Mieux comprendre la Cour Pénale Internationale, La Haye, Pays Bas.

Esmein V., Le prix d'une espérance de vie, D. Chronique, 1962.

Fofe Djofia Malewa, Les caractéristiques victimocentriques du droit pénal traditionnel, Revue de la Faculté de Droit, numéro spécial ,1ère année, 1998, Kinshasa, 1998.

Fonds au profit des victimes, Assistance et réparations, réalisations, enseignements tirés et évolution, Rapport sur l'état d'avancement des programmes, Septembre 2015.

Gasigwa H., Le droit et la morale dans l'indemnisation des dommages corporels, Bukavu, CERUKI, 2000.

Geneviève Viney, Le déclin de la responsabilité individuelle, Paris, 1965.

Grégory Mestre, La responsabilité civile à l'épreuve de l'analyse économique du droit, Paris, LGDJ, 2005.

Guidon M., Le préjudice esthétique, Paris, LGDJ, 2006.

ICC-ASP/1/Res. 6 portant création d'un fonds au profit des victimes de crimes relevant de la compétence de la Cour et de leurs familles, adoptée par consensus, à la séance plénière, le 9 septembre 2002.

ICC-ASP/3/Res. 7, Création du Secrétariat du fonds d'affectation spéciale au profit des victimes, adoptée le 10 Septembre 2004.

ICC, Résolution ICC-ASP/4/Res. 3 relative au Règlement du fonds d'affectation spéciale au profit des victimes, adoptée le 3 décembre 2005.

Jeangene V., Réparer l'irréparable : Les réparations aux victimes devant la Cour pénale internationale, Presses Universitaires de France, Paris, 2009.

Kangulumba Mbambi V., Indemnisation des victimes des accidents de circulation et assurance de responsabilité civile automobile. Etude de droit comparé belge et congolais, Thèse de doctorat, Louvain-La-Neuve, Bruylant, 2002.

Kihangi Bindu K., «L'institution d'une juridiction pénale internationale pour les crimes internationaux commis en République Démocratique du Congo : Une nécessité de justice et de paix durable ", Revue Africaine de la Démocratie et de la Gouvernance (RADG), Vol. 2 ? N $1 \& 2,2015$.

Kihangi Bindu Kennedy, Etat des lieux de l'action de la Cour Pénale Internationale dans la lutte contre l'impunité en RDC, Programme des Nations Unies de Perfectionnement en Droit International, inédit, Juillet / Août, à la Haye/ Pays Bas, inédit, 2010.

Kihangi Bindu Kennedy, « Problématique du concept de justice et paix dans la poursuite des crimes graves commis à l'Est de la RDC », Recueil des actes des journées scientifiques, Les 10 ans de la Cour Pénale Internationale : Bilan et perspectives, Kinshasa, 23 au 25 octobre 2012.

Lambert Y., Des préjudices réfléchis nés de l'atteinte à la vie ou à l'intégrité corporelle, LGDJ, 1990.

Letourneau P., La Responsabilité civile, Paris, Dalloz, 1982.

Loi n 023/2002 du 18 Novembre 2002 portant Code de justice militaire, 44ème année, n spécial, 20 mars 2003 , telle que modifiée à ce jour en République Démocratique du Congo.

Marty G., « De la Relation de cause à effet comme condition de la responsabilité civile : Etude comparative des conceptions allemande, anglaise, et française », Revue Trimestrielle de Droit Civile, 1939.

Montanier J., L'incidence des prédispositions de la victime sur la causalité du dommage, Grenoble, 1981. 
Mubalama Zibona, J.C. Responsabilité, solidarité, sécurité. A la recherche d'un mécanisme de socialisation des risques liés à la contamination par le virus du SIDA en Afrique sub-saharienne, Thèse de doctorat, Université Catholique de Louvain, 2005.

Mushiata G. \& Parmar S., Déni de justice : Les Victimes de crimes graves ne reçoivent pas les réparations ordonnées par la Cour de justice en République Démocratique du Congo, Editions ICTJ, Kinshasa, 2013.

Nguyen J., "L'influence des prédispositions de la victime sur l'obligation à réparation du défendeur à l'action en responsabilité », Revue trimestrielle de Droit civil, 1976.

Nyabirungu R., Droit international pénal : Crimes contre la paix et la sécurité de l'humanité, DES, Kinshasa, 2013.

Patrick Tshibuyi wa Tshibuyi, Module de sensibilisation sur la Cour Pénale Internationale, Université Libre des Pays des Grands Lacs, Faculté de droit, Goma, Inédit, 2009.

Philippe Xavier, «Vers une reconnaissance accrue de la place de la victime dans le procès pénal international ", l'actualité de la justice pénale internationale, 2007.

PNUD, Etude sur la réparation des préjudices subis par les victimes des crimes contre la paix et la sécurité de I'humanité, y compris les violences sexuelles, Inédit, 2018.

Pradel J., Droit pénal comparé, Dalloz, Paris, 2008.

Redress, Ending torture, Seeking justice for Survivor : Faire avancer la réparation à la CPI : Recommandations, Novembre 2016.

Redress Trust, Ending torture, Seeking justice for Survivor : Ne plus perdre de temps, la mise en œuvre des réparations pour les victimes devant la Cour Pénale Internationale, Janvier 2019.

Règlement de Procédure et de Preuve, New York, 2002.

Résolution de l'Assemblée Générale des Nations Unies, A/RES/60/147 du 16 Décembre 2005.

Résolution du Conseil de Sécurité des Nations Unies 827 du 25 mai 1993 créant le Tribunal Pénal International pour l'ex. Yougoslavie.

Résolution du Conseil de Sécurité des Nations Unies 955 le 8 novembre 1994 créant le Tribunal Pénal International pour le Rwanda.

Ripert G., Le Prix de la douleur, Paris, Chronique, 1948.

Savatier R., Traité de la responsabilité civile en droit français, Paris, LGDJ, 2000.

Schimitt D., Les fonds internationaux en faveur des victimes de violations de droits de l'homme et du droit international humanitaire, Thèse de doctorat, Université Panthéon-Sorbonne-Paris I, 2016.

Starck Boris, « Domaine et fondement de la responsabilité sans faute », Revue trimestrielle de droit civil, 1958.

Statut de Rome de la Cour Pénale Internationale de 1998

Telomono M., « Repenser la téléologie et les modalités de la réparation en droit civil Congolais : De la réparation intégrale du dommage à la réparation adéquate du lien social ", Annales de la Faculté de Droit de l'Université de Kinshasa, Kinshasa, DES, 2014.

Terré F. et alii, Droit civil : Les obligations, 7ème édition, Paris, Dalloz, 1999.

Thomas Besse, "Les droits des victimes devant la justice pénale internationale : Entre certitudes et doutes ", Sources ouvertes, Paris, 2013.

Vidal J., L'arrêt de la Chambre mixte du 27 Février 1970, Le droit à réparation de la concubine et le concept de dommage réparable, JCP, 1971.

Vidal J., La réparation du préjudice issu de la perte de chance : Cas de la mort d'un des conjoints, LGDJ, Paris, 1980.

Le Procureur c. Mathieu Ngudjolo, ICC-01/04-02/12, ICC-PIDS-CIS-DRC-06-006/15-tFra, https://www.icc-cpi. int/CaselnformationSheets/ChuiFra.pdf (visité le 29/05/ 2020). 
Le Procureur c. Thomas Lubanga Dyilo, ICC-01/04-01/06, ICC-PIDS-DRC-01-016/17_Fra, https://www.icc-cpi. int/CaselnformationSheets/LubangaFra.pdf (visité, le 29/05/2020).

Le Procureur c. Germain Katanga, ICC-01/04-01/07, ICC-014/18_Fra, https://www.icc-cpi.int/CaselnformationSheets/KatangaFra.pdf (visité le 29/05/2020).

Le Procureur c. Jean Pierre Bemba Gombo, ICC-01/05-01/08, ICC-PIDS-CAR-01-020/18_Fra,

https://www.icc-cpi.int/CaselnformationSheets/BembaFra.pdf (visité le 29/05/2020).

Le Procureur c. Ahmad Al Faqi Al Mahdi, ICC-01/12-01/15, ICC-PIDS-CIS-MAL-01-08/16_Fra, https://www.icc-cpi.int/mali/al-mahdi?ln=fr, (visité le 29/05/2020).

Le Procureur c. William Samoei Ruto et Joshua Arap Sang, ICC-01/09-01/11, ICC-PIDS-CIS-KEN-01-12/14_Fra, https://www.icc-cpi.int/CaselnformationSheets/RutoSangFra.pdf (visité le 29/05/2020).

Le Procureur $c$. Thomas Lubanga, Chambre de Première Instance I, Décision Fixant les principes et procédures applicables en matière de réparations, ICC-01/04-01/06 du 7 août 2012, ICC-01/04-01/06-2904-tFRA, le 19 février 2013.

Le Procureur c. Thomas Lubanga Dyilo, Chambre d'appel, Ordonnance de réparation modifiée, du 3 mars 2015, ICC-01/04-01/06-3129-AnxA-tFRA, le 1er août 2016.

Le Procureur c. Thomas Lubanga Dyilo, Chambre de Première Instance II, Rectificatif de la " Décision fixant le montant des réparations auxquelles Thomas Lubanga Dyilo est tenu », version publique expurgée, ICC-01/0401/06 du 21 décembre 2017, ICC-01/04-01/06-3379-Red-Corr du 21 décembre 2017.

Le Procureur c. Germain Katanga, Chambre de Première Instance II, Ordonnance de réparation, ICC-01/04-01/073728 du 24 mars 2017 ;

Le Procureur c. Ahmad Al Faqi Al Mahdi, Ordonnance de réparation, ICC-01/12-01/15-236-tFRA du 17 août 2017, VII, PP. 66/67.

Motoo Noguchi M., Rapport à l'Assemblée des Etats parties sur les activités et les projets du conseil de direction du Fonds d'affectation spéciale au profit des victimes pour la période du 1er juillet 2012 au 30 Juin 2013, Paragraphes 39 et 46, https://asp.icc-cpi.int/iccdocs/asp_docs/ASP15/ICC-ASP-15-14-FRA.pdf

Le Conseil de direction du Fonds au profit des victimes affirme que la justice réparatrice prévue par le Statut de Rome revêt une importance incontestable pour les victimes, Déclaration du 14 Septembre 2018, https://www.icc-cpi.int/Pages/item.aspx?name=180914-stat-tfv\&ln=fr, (visité le 5 mai 2020).

Joachim Unegi, Directeur de la Radio Colombe de Mahagi (180 km au nord de Bunia, à la frontière avec l'Ouganda), Les victimes des crimes craignent pour l'effectivité de la réparation suite à la libération de Thomas Lubanga, 25 mars 2020, https://french.lubangatrial.org/2020/03/25/les-victimes-des-crimes-craignent-pour-leffectivite-de-la-reparation-suite-a-la-liberation-de-thomas-lubanga/

Recebido/Received: 21.06.2020.

Aprovado/Approved: 08.08.2020. 